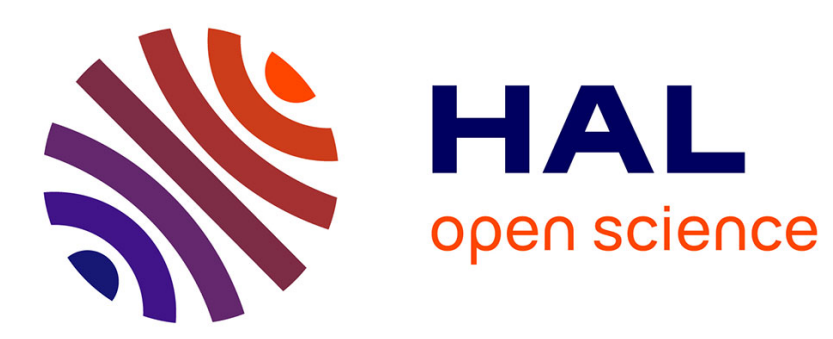

\title{
Electromechanical response of multilayered piezoelectric BaTiO3/PZT-7A composites with wavy architecture
}

\author{
Wenqiong Tu, Qiang Chen
}

\section{To cite this version:}

Wenqiong Tu, Qiang Chen. Electromechanical response of multilayered piezoelectric BaTiO3/PZT7A composites with wavy architecture. Journal of Intelligent Material Systems and Structures, 2021, pp.1045389X2098388. 10.1177/1045389x20983887 . hal-03144463

\section{HAL Id: hal-03144463 \\ https://hal.science/hal-03144463}

Submitted on 17 Feb 2021

HAL is a multi-disciplinary open access archive for the deposit and dissemination of scientific research documents, whether they are published or not. The documents may come from teaching and research institutions in France or abroad, or from public or private research centers.
L'archive ouverte pluridisciplinaire HAL, est destinée au dépôt et à la diffusion de documents scientifiques de niveau recherche, publiés ou non, émanant des établissements d'enseignement et de recherche français ou étrangers, des laboratoires publics ou privés. 


\title{
Electromechanical response of multilayered piezoelectric $\mathrm{BaTiO}_{3} /$ PZT-7A composites with wavy architecture
}

\author{
Wenqiong Tu' and Qiang Chen ${ }^{2}$ (D)
}

\begin{abstract}
Electromechanical laminated composites with piezoelectric phases are increasingly being explored as multifunctional materials providing energy conversion between electric and mechanical energies. The current work explores thus-far undocumented combined microstructural effects of amplitude-to-wavelength ratio, volume fraction, poling direction of piezoelectric phases on both the homogenized properties and localized stress/electric field distributions in multilayered configurations under fully coupled electro-mechanical loading. In particular, the Multiphysics Finite-Volume Direct Averaging Micromechanics (FVDAM) and its counterpart, an in-house micromechanical multiphysics finite-element model, are utilized to investigate the homogenized and localized responses of wavy multilayered piezoelectric $\mathrm{BaTiO}^{3} /$ PZT-7A architectures. These two methods generate highly agreeable results. Moreover, we critically examine the convergence of the finite-volume and finite element-based approaches via the Average Stress Theorem and Average Electric Displacement Theorem. The comparison shows the finite volume-based approach possesses a better numerical convergence. This study illustrates the FVDAM's ability toward the analysis and design of engineered multilayered piezoelectric materials with wavy architecture.
\end{abstract}

\section{Keywords}

Multilayered wavy composites, homogenization, piezoelectric effect, finite-volume theory, finite-element theory

\section{Introduction}

As an essential component of sensors, actuators, transducers, and energy harvesting devices under intensive research and development (Li et al., 2019; Liu et al., 2018; Na and Baek, 2018), the piezoelectric material possesses the capability to convert mechanical energy to electric energy through direct piezoelectric effect or vis-à-vis through the inverse piezoelectric effect. While monolithic piezoelectric materials have several limitations, such as brittleness, limited range of coupled properties, and pronounced directionality (Kar-Gupta and Venkatesh, 2005, 2007), the composite piezoelectric materials offer a promising solution toward the above issues (Kar-Gupta and Venkatesh, 2013; Kar-Gupta et al., 2008; Sun and Kim, 2010).

To use and design the composite piezoelectric materials effectively, it's indispensable to have certain micromechanics techniques which can take into account the piezoelectric effect under thermal, electric, and mechanical loads in an accurate and efficient manner. In past decades, the classical micromechanics models, such as
Mori-Tanaka, composite cylinder/sphere assemblage, and three-phase models, which are based on single fiber embedded in another media, have been extensively developed to calculate the homogenized properties of piezoelectric composites (Chatzigeorgiou et al., 2019; Jiang and Cheung, 2001; Li and Dunn, 1998; Xiao et al., 2016; Yu Li and Dunn, 2001). However, those models are microstructural detail-free models which only estimate the homogenized properties and do not predict the accurate internal stress/strain, as well as electric displacement distributions. To fully understand the performance of piezoelectric materials and facilitate their applications in the real world, it's important to

\footnotetext{
'School of Automotive and Traffic Engineering, Jiangsu University, Zhenjiang, China

${ }^{2}$ LEM3-UMR 7239 CNRS, Arts et Métiers ParisTech Metz, Metz, France
}

\section{Corresponding author:}

Qiang Chen, LEM3-UMR 7239 CNRS, Arts et Métiers ParisTech Metz, 4 Rue Augustin Fresnel, Metz 57078, France.

Email: qiang.chen@ensam.eu 
develop effective computational micromechanics models for piezoelectric composites which can both predict accurate homogenized and local responses. The finite element approach is the major approach to create micromechanics models. However, in order to obtain the homogenized and localized responses of unidirectional piezoelectric composites, a three-dimensional unit cell analysis needs to be conducted in the commercial software, such as Abaqus, and the periodic boundary conditions of the nodal displacements and electric potential need to be enforced explicitly during the model creation process (Chen et al., 2018a). To overcome the above computational inefficiency and applicational cumbrousness, a fully anisotropic twodimensional Q8-type multiphysics quadratic element has been constructed under the generalized plane strain constraint (Chen and Wang, 2020). Hence, a full set of homogenized moduli can be generated directly without using the time-consuming 3D analysis of unit cell problems. Meanwhile, the parametric multiphysics Finite Volume Direct Averaging Micromechanics has proven to be an effective tool to obtain an accurate homogenized and local responses of unidirectional piezoelectric composites with the added advantages of stability and efficiency (Chen and Wang, 2020).

As an important type of piezoelectric composites, the flat multilayered piezoelectric composites have been studied extensively. For instance, the multilayered piezocomposites are utilized to yield high hydrostatic piezoelectric response, to satisfy the demanding requirements of clinic ultrasound imaging (Cannata et al., 2006), and to improve the shear piezoelectric actuation of beams and annular plates via an off-axis lamination design (Dubey and Panda, 2019; Panda and Kumar Dubey, 2020), as well as to create effective energy harvesting devices via parallel links (Asano et al., 2020) or a stacking design (Kim et al., 2020). Further, to better understand the relation between the constituent material properties and the overall response of the layered system which would enable the tailoring of composite system's performance, the electromechanical response and homogenized material properties have been extensively investigated via both analytical and numerical approaches by various researchers (Camarena and $\mathrm{Yu}$, 2019; Challagulla and Venkatesh, 2009; Kar-Gupta and Venkatesh, 2013; Kim et al., 2009; Wang et al., 2009; Zhang et al., 2019).

In contrast with the well-studied regular flat multilayered piezoelectric composites, research work on the piezoelectric multilayers with wavy architectures is limited but shows great potential. Most recently, the piezoelectric multilayers with wavy architectures are employed to develop an advanced intelligent acoustic emission (AE) and mechanical impedance hybrid sensor (Feng and Huang, 2013; Feng et al., 2009), an efficient wearable energy harvesting system with high stretchability (Feng et al., 2011; Qi et al., 2011) and a sandwich-type piezoelectric energy harvester powered by human walking (Han et al., 2016; Singh et al., 2018). It is worth mentioning that the mechanical behaviors of multilayers with wavy architectures have been extensively studied, including thermal-elastic behavior (Khatam and Pindera, 2009b), plasticity (Katz et al., 2015; Khatam and Pindera, 2009a), finite deformation ( $\mathrm{Tu}$ and Pindera, 2013). The above work illustrates wavy multilayers' capability in achieving excellent toughness and mimics the biomaterial's performance by designing the wavy microstructures and constituent properties. To fully exploit the potential of the wavy multilayered piezoelectric composites, it is essential to understand the relation between the constituent's material properties, material microstructures, and the macro electromechanical behavior of the wavy layered system. However, to the best knowledge of the authors, the homogenized and local responses of piezoelectric multilayers with wavy architecture have never been investigated.

The main objective of the current paper is to understand the effects of amplitude-to-wavelength ratio, volume fraction, and the poling direction of constituent on both the homogenized properties and local stress/electric field distributions of the wavy multilayered piezoelectric $\mathrm{BaTiO}^{3} / \mathrm{PZT}-7 \mathrm{~A}$ architectures. Secondly, the paper critically examines the accuracy of finite volume and finite element-based micromechanics approaches. The remainder of the paper is organized as follows: Section 2 describes the theoretical framework of the multiphysics FVDAM, including the homogenization framework, generalized local stiffness construction, generalized stiffness matrix assembly, and homogenization. Section 3 validates the multiphysics FVDAM against an analytical solution found in the literature and then focuses on the numerical results of the wavy multilayered piezoelectric $\mathrm{BaTiO}^{3} / \mathrm{PZT}-7 \mathrm{~A}$ architectures, demonstrating the effects of amplitude-towavelength ratio, volume fraction, the poling direction of constituent on both the homogenized properties and local stress/electric field distributions. In Section 4, the accuracy of FVDAM and finite-element based multiphysics micromechanics are accessed based on the average stress and electric displacement theory. Discussions and future work are described in Section 5. The summary and conclusions are presented in Section 6.

\section{Multiphysics FVDAM}

The details of the mechanical version of the finitevolume direct averaging micromechanics approach have been presented in a sequence of papers (Cavalcante and Pindera, 2016; Cavalcante et al., 2012; Chen and Pindera, 2020; Chen et al., 2018b). The FVDAM with coupled thermo-electro-elastic capability was developed recently by Chen et al. (2018a) for periodic materials undergoing infinitesimal deformations in order to 
generate higher fidelity of stress and electric fields relative to the classical micromechanics theories and finiteelement techniques. For a piezoelectric material, the generalized Hooke's Law reads:

$$
\begin{aligned}
\sigma_{i j} & =C_{i j k l} \varepsilon_{k l}-e_{k i j} E_{k} \\
D_{i} & =e_{i k l} \varepsilon_{k l}+\kappa_{i k} E_{k}
\end{aligned}
$$

where $\sigma_{i j}$ and $D_{i}$ are the stress and electric displacement components, respectively, $\varepsilon_{i j}$ and $E_{k}$ are the strain and electric field components, respectively. $C_{i j k l}, e_{i k l}$, and $\kappa_{i k}$ denote the stiffness, piezoelectric coefficients, and dielectric coefficients, respectively.

Equation (1) can be expressed in matrix and vector notations for an easier implementation into the FVDAM analysis:

$$
\left[\begin{array}{l}
\boldsymbol{\sigma} \\
\mathbf{D}
\end{array}\right]=\mathbf{Z}\left[\begin{array}{c}
\boldsymbol{\varepsilon} \\
-\mathbf{E}
\end{array}\right]
$$

where $\boldsymbol{\sigma}=\left[\begin{array}{llllll}\sigma_{11} & \sigma_{22} & \sigma_{33} & \sigma_{23} & \sigma_{13} & \sigma_{12}\end{array}\right]^{\mathrm{T}}, \quad \boldsymbol{\varepsilon}=\left[\begin{array}{lll}\varepsilon_{11} & \varepsilon_{22} & \varepsilon_{33}\end{array}\right.$ $\left.\begin{array}{lll}2 \varepsilon_{23} & 2 \varepsilon_{13} & 2 \varepsilon_{12}\end{array}\right]^{\mathrm{T}}, \quad \mathbf{D}=\left[\begin{array}{lll}D_{1} & D_{2} & D_{3}\end{array}\right]^{\mathrm{T}}, \quad \mathbf{E}=\left[\begin{array}{lll}E_{1} & E_{2} & E_{3}\end{array}\right]^{\mathrm{T}}$, and $\mathbf{Z}=\left[\begin{array}{cc}\mathbf{C} & \mathbf{e}^{\mathrm{T}} \\ \mathbf{e} & -\mathbf{K}\end{array}\right]$. To accommodate the general anisotropic properties of poled piezoceramics, the submatrices of the generalized stiffness matrix are defined in this work as:

$$
\begin{aligned}
\mathbf{C} & =\left[\begin{array}{llllll}
C_{11} & C_{12} & C_{13} & C_{14} & C_{15} & C_{16} \\
C_{12} & C_{22} & C_{23} & C_{24} & C_{25} & C_{26} \\
C_{13} & C_{23} & C_{33} & C_{34} & C_{35} & C_{36} \\
C_{14} & C_{24} & C_{34} & C_{44} & C_{45} & C_{46} \\
C_{15} & C_{25} & C_{35} & C_{45} & C_{55} & C_{56} \\
C_{16} & C_{26} & C_{36} & C_{46} & C_{56} & C_{66}
\end{array}\right], \\
\mathbf{e} & =\left[\begin{array}{llllll}
e_{11} & e_{12} & e_{13} & e_{14} & e_{15} & e_{16} \\
e_{21} & e_{22} & e_{23} & e_{24} & e_{25} & e_{26} \\
e_{31} & e_{32} & e_{33} & e_{34} & e_{35} & e_{36}
\end{array}\right], \\
\mathbf{\kappa} & =\left[\begin{array}{lll}
\kappa_{11} & \kappa_{12} & \kappa_{13} \\
\kappa_{12} & \kappa_{22} & \kappa_{23} \\
\kappa_{13} & \kappa_{23} & \kappa_{33}
\end{array}\right] .
\end{aligned}
$$

\section{I. Homogenization framework}

The multiphysics FVDAM framework is a simplified version of the mathematical homogenization theory. The latter is based on a systematic asymptotic analysis of periodic media whose response is characterized by governing differential equations with periodically varying coefficients that reflect the spatial variation of the microstructures (Charalambakis, 2010; Yang et al., 2020; Zhu et al., 2020). The mathematical homogenization theory provides a consistent framework for taking into account the effect of macroscopic strain and electric field variations in a periodic material whose microstructural scale is characterized by the small parameter $\mathbf{s}$. Within this framework, the macroscopic and local variations of displacements and electric potentials are described using the global and local scales $\boldsymbol{x}$ and $\boldsymbol{y}$, respectively, connected through the small parameter $\boldsymbol{s}=\boldsymbol{y} / \boldsymbol{x}$. The operator $\partial / \partial x_{i}$ in the governing differential equations is then replaced by $\frac{\partial}{\partial x} \rightarrow \frac{\partial}{\partial x}+\frac{1}{s} \frac{\partial}{\partial y}$ which separates the governing differential equations into different orders of $\mathbf{s}$ or scales.

Consistent with the concept of scale separation, a multiscale displacement $u_{i}^{(\mathbf{s})}(\mathbf{x}, \mathbf{y})$ and electric potential $\alpha^{(\mathbf{s})}(\mathbf{x}, \mathbf{y})$ field representation based on the small parameter $\mathbf{s}$,

$$
\begin{aligned}
& u_{i}^{(\mathrm{s})}(\mathbf{x}, \mathbf{y})=u_{i}^{(0)}(\mathbf{x}, \mathbf{y})+\varsigma u_{i}^{(1)}(\mathbf{x}, \mathbf{y})+O\left(\mathrm{~s}^{2}\right) \\
& \alpha^{(\mathrm{s})}(\mathbf{x}, \mathbf{y})=\alpha^{(0)}(\mathbf{x}, \mathbf{y})+\varsigma \alpha^{(1)}(\mathbf{x}, \mathbf{y})+O\left(\mathrm{~s}^{2}\right)
\end{aligned}
$$

is used to separate the governing differential equations into equations of different scale orders.

In the finite-volume homogenization theory, the displacement and electric potential field in each phase of the microstructure is partitioned into average and fluctuating contributions dependent on the global and local coordinates, $\boldsymbol{x}=\left(x_{1}, x_{2}, x_{3}\right)$ and $\boldsymbol{y}=\left(y_{1}, y_{2}, y_{3}\right)$, respectively, without considering the higher-order terms:

$$
\begin{aligned}
& u_{i}^{(q)}(\mathbf{x}, \mathbf{y})=\bar{\varepsilon}_{i j} x_{j}+u_{i}^{\prime(q)}(\mathbf{y}) \\
& \alpha^{(q)}(\mathbf{x}, \mathbf{y})=-\bar{E}_{j} x_{j}+\alpha^{\prime(q)}(\mathbf{y})
\end{aligned}
$$

where $\bar{\varepsilon}_{i j}$ and $\bar{E}_{j}$ are macroscopic strain and electric field. $u_{i}^{\prime(q)}(i=1,2,3)$ and $\alpha^{\prime(q)}$ are displacement and electric potential fluctuations.

Herein, we limit our analysis to multilayered wavy composites with continuous reinforcements whose response may be characterized by periodically repeating material microstructure with two-dimensional periodicity, Figure 1(a). The unit cell is discretized into quadrilateral subvolumes designated by the index $(q)$, whose location is specified by the subvolume vertices $\left(y_{2}^{(i, q)}, y_{3}^{(i, q)}\right)$ referred to the fixed RUC coordinate system. The qth subvolume is generated by mapping the square-sided subvolume in the reference coordinates $(\eta-\xi)$ onto a quadrilateral subvolume resident in the actual microstructure, Figure 1(b), using the Q4-type mapping function:

$$
y_{i}^{(q)}(\mathbf{x}, \mathbf{y})=\sum_{j=1}^{4} N_{j}(\eta, \xi) y_{i}^{(j, q)}, \quad i=2,3
$$

where $N_{j}(\eta, \xi)$ is the shape function given by: 

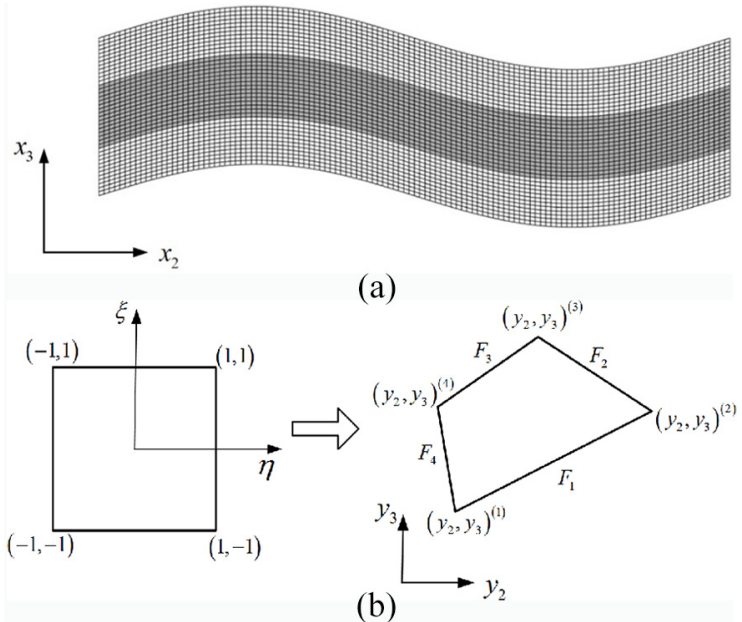

Figure I. (a) Discretization of repeating unit cell of multilayered piezoelectric composites with sinusoidal wavy architecture and (b) mapping transformation in parametric FVDAM.

$$
\begin{aligned}
& N_{1}(\eta, \xi)=\frac{1}{4}(1-\eta)(1-\xi), N_{2}(\eta, \xi)=\frac{1}{4}(1+\eta)(1-\xi) \\
& N_{3}(\eta, \xi)=\frac{1}{4}(1+\eta)(1+\xi), N_{4}(\eta, \xi)=\frac{1}{4}(1-\eta)(1+\xi)
\end{aligned}
$$

\subsection{Generalized local stiffness construction}

The fluctuating displacements $u_{i}^{\prime(q)}(i=1,2,3)$ and electric potentials $\alpha^{\prime(q)}$ in the qth subvolume are approximated using the following polynomial expansion in the reference coordinates $(\eta-\xi)$ :

$$
\begin{aligned}
{u_{i}^{\prime}}_{i}^{(q)}= & W_{i(00)}^{(q)}+\eta W_{i(10)}^{(q)}+\xi W_{i(01)}^{(q)} \\
& +\frac{1}{2}\left(3 \eta^{2}-1\right) W_{i(20)}^{(q)}+\frac{1}{2}\left(3 \xi^{2}-1\right) W_{i(02)}^{(q)} \\
\alpha^{\prime(k)}= & W_{4(00)}^{(q)}+\eta W_{4(10)}^{(q)}+\xi W_{4(01)}^{(q)} \\
& +\frac{1}{2}\left(3 \eta^{2}-1\right) W_{4(20)}^{(q)}+\frac{1}{2}\left(3 \xi^{2}-1\right) W_{4(02)}^{(q)}
\end{aligned}
$$

where $W_{i(.)}^{(q)}$ are the unknown microvariables associated with each subvolume, which ensures that $\bar{\varepsilon}_{11}=\varepsilon_{11}^{(q)}$ and $\bar{E}_{1}=E_{1}^{(q)}$ due to the continuous reinforcement in $x_{1}$ direction.

The employed two-scale expansion of displacements and electric displacements produces local strain and electric field in the form:

$$
\begin{aligned}
& \varepsilon_{i j}^{(q)}=\bar{\varepsilon}_{i j}+\varepsilon_{i j}^{\prime(q)}=\bar{\varepsilon}_{i j}+\frac{1}{2}\left(\frac{\partial u_{i}^{\prime}}{\partial y_{j}}+\frac{\partial u_{j}^{\prime}}{\partial y_{i}}\right)^{(q)} \\
& E_{i}^{(q)}=\bar{E}_{i}+E_{i}^{\prime(q)}=\bar{E}_{i}-\frac{\partial \alpha^{\prime(q)}}{\partial y_{i}}
\end{aligned}
$$

Evaluating surface-averaged interfacial displacements and electric potentials for the qth subvolume in the reference coordinates produces the surface-averaged displacements and electric potentials (the subscript $q$ is omitted for convenience), which provides the fundamental unknowns for the parametric multiphysics FVDAM:

$$
\begin{array}{lll}
\hat{u}_{i}^{\prime(1,3)} & =\frac{1}{2} \int_{-1}^{+1} u_{i}^{\prime}(\eta, \mp 1) \mathrm{d} \eta & \hat{u}_{i}^{\prime(2,4)}=\frac{1}{2} \int_{-1}^{+1} u_{i}^{\prime}( \pm 1, \xi) \mathrm{d} \xi \\
\hat{\alpha}^{\prime(1,3)}=\frac{1}{2} \int_{-1}^{+1} \alpha^{\prime}(\eta, \mp 1) \mathrm{d} \eta & \hat{\alpha}^{\prime(2,4)}=\frac{1}{2} \int_{-1}^{+1} \alpha^{\prime}( \pm 1, \xi) \mathrm{d} \xi
\end{array}
$$

The first- $\left(W_{i(10)}^{(q)}, W_{i(01)}^{(q)}\right)$ and second- $\left(W_{i(20)}^{(q)}, W_{i(02)}^{(q)}\right)$ order coefficients are explicitly expressed in terms of surface-averaged displacements and electric potentials as well as the zeroth-order coefficients $\left(W_{i(00)}^{(q)}\right)$ upon using the definition in equation (9). The four remaining equations for the determination of the zeroth-order coefficients are introduced by satisfying the stress equilibrium equation and conservation equations in an average sense within each subvolume:

$$
\frac{1}{V_{q}} \int_{V_{q}} \frac{\partial \sigma_{i j}}{\partial x_{j}} d V=0, \quad \frac{1}{V_{q}} \int_{V_{q}} \frac{\partial D_{i}}{\partial x_{i}} d V=0
$$

Evaluating the surface-averaged interfacial tractions and normal electric displacements in terms of surfaceaveraged mechanical displacements and electric potentials produces a system of generalized local stiffness equations which can be symbolically expressed as:

$$
\hat{\mathbf{Y}}^{(q)}=\mathbf{K}^{(q)} \hat{\mathbf{U}}^{(q)}+\mathbf{N}^{(q)} \mathbf{Z}^{(q)} \overline{\mathbf{X}}
$$

where

$$
\hat{\mathbf{Y}}^{(q)}=\left[\begin{array}{llll}
\hat{\mathbf{p}}^{(1)} & \hat{\mathbf{p}}^{(2)} & \hat{\mathbf{p}}^{(3)} & \hat{\mathbf{p}}^{(4)}
\end{array}\right]^{(q)^{\mathrm{T}},}, \quad \hat{\mathbf{p}}^{(p, q)}=
$$
$\left[\begin{array}{llll}\hat{t}_{1} & \hat{t}_{2} & \hat{t}_{3} & \hat{f}\end{array}\right]^{(p, q) \mathrm{T}}, t_{i}=\sigma_{i j} n_{j}, f=D_{i} n_{i}$, and $\left[\begin{array}{lll}n_{1} & n_{2} & n_{3}\end{array}\right]$ are the unit normal vectors that define the orientation of each face of a quadrilateral subvolume. $\hat{\mathbf{U}}^{(q)}=\left[\begin{array}{llll}\hat{\mathbf{u}}^{(1)} & \hat{\mathbf{u}}^{\prime(2)} & \hat{\mathbf{u}}^{(3)} & \hat{\mathbf{u}}^{(4)}\end{array}\right]^{(q) \mathrm{T}}, \quad \hat{\mathbf{u}}^{(p, q)}=$ $\left[\begin{array}{llll}\hat{u}_{1}^{\prime} & \hat{u}_{2}^{\prime} & \hat{u}_{3}^{\prime} & \hat{\alpha}^{\prime}\end{array}\right]^{(p, q)^{\mathrm{T}},} \quad \overline{\mathbf{X}}=\quad\left[\begin{array}{llll}\bar{\varepsilon}_{11} & \bar{\varepsilon}_{22} & \bar{\varepsilon}_{33} & 2 \bar{\varepsilon}_{23}\end{array}\right.$ $\left.2 \bar{\varepsilon}_{13} \quad 2 \bar{\varepsilon}_{12} \quad-\bar{E}_{1} \quad-\bar{E}_{2} \quad-\bar{E}_{3}\right]^{\mathrm{T}}, \quad \mathbf{N}^{(q)}=\left[\begin{array}{ll}\mathbf{n}^{(1)} & \mathbf{n}^{(2)}\end{array}\right.$ $\left.\mathbf{n}^{(3)} \mathbf{n}^{(4)}\right]^{(q) \mathrm{T}}$,

$\mathbf{n}^{(p, q)}=\left[\begin{array}{ccccccc}0 & 0 & 0 & n_{3} & n_{2} & 0 & 0 \\ n_{2} & 0 & n_{3} & 0 & 0 & 0 & 0 \\ 0 & n_{3} & n_{2} & 0 & 0 & 0 & 0 \\ 0 & 0 & 0 & 0 & 0 & n_{2} & n_{3}\end{array}\right]^{(p, q)} . \mathbf{Z}^{(q)} \quad$ is the generalized local stiffness matrix of the $q$ th subvolume: $\mathbf{Z}^{(q)}=\left[\begin{array}{cc}\mathbf{C} & \mathbf{e}^{\mathrm{T}} \\ \mathbf{e} & -\mathbf{K}\end{array}\right]^{(q)}$. The generalized local stiffness matrices $\mathbf{K}^{(q)}$ given explicitly by the subvolume 
geometry and material properties can be found in reference (Chen et al., 2018a).

\subsection{Generalized stiffness matrix assembly}

The unknown interfacial surface-averaged displacements and electric potentials are determined by solving a global system of equations generated by first imposing traction and electric displacement continuity conditions at common faces of adjacent subvolumes, followed by direct enforcement of fluctuating mechanical displacement and electric potential continuity conditions. The resulting global system of equations can be symbolically represented by:

$$
\mathbb{K} \hat{U}^{\prime}=\Delta \mathbb{Z} \overline{\mathbf{X}}
$$

where $\mathbb{K}$ denotes the generalized global stiffness matrix, the vector $\hat{\mathbb{U}}^{\prime}$ is comprised of all the unknown interfacial mechanical displacements and electric potentials. The matrix $\Delta \mathbb{Z}$ represents the differences in the generalized local stiffness matrices of adjacent subvolumes.

\subsection{Homogenization}

The solution of the global system of equations, equation (12), yields the fluctuating displacement components and electric potentials, hence the local strains and electric fields in equation (8). The generalized electroelastic concentration matrices relating averaged subvolume strains and electric fields and their applied counterparts (Hill, 1963), also noted as localization relations, are established as:

$$
\left[\begin{array}{l}
\overline{\boldsymbol{\varepsilon}} \\
\overline{\mathbf{E}}
\end{array}\right]^{(q)}=\mathbf{A}^{(q)}\left[\begin{array}{c}
\overline{\boldsymbol{\varepsilon}} \\
\overline{\mathbf{E}}
\end{array}\right]
$$

where the matrices $\mathbf{A}^{(q)}$ can be determined by successive imposition of unit macroscopic strain or electric field at a time and then solving the corresponding boundary value problems to obtain average subvolume strains and electric fields at the specified loading condition.

The homogenized stresses and electric displacements for the unit cell of a volume $V$ are expressed as a weighted sum of the averaged stresses and electric displacements over all elements:

$$
\begin{aligned}
& \overline{\boldsymbol{\sigma}}=\frac{1}{V} \int \boldsymbol{\sigma}(\mathbf{x}) \mathrm{d} V=\frac{1}{V} \sum_{q=1}^{N_{q}} \int_{V_{q}} \boldsymbol{\sigma}^{(q)}(\mathbf{x}) \mathrm{d} V^{(q)}=\sum_{q=1}^{N_{q}} v^{(q)} \overline{\boldsymbol{\sigma}}^{(q)} \\
& \overline{\mathbf{D}}=\frac{1}{V} \int \mathbf{D}(\mathbf{x}) \mathrm{d} V=\frac{1}{V} \sum_{q=1}^{N_{q}} \int_{V_{q}} \mathbf{D}^{(q)}(\mathbf{x}) \mathrm{d} V^{(q)}=\sum_{q=1}^{N_{q}} v^{(q)} \overline{\mathbf{D}}^{(q)}
\end{aligned}
$$

where $V^{(q)}$ is the volume of the $q$ th subvolume, and $v^{(q)}=V^{(q)} / V$ is the corresponding volume fraction. Taking the localization relations in the expression for the averaged composite stresses and electric displacements, in conjunction with the element-averaged constitutive relation of $q$ th element, we obtain the global (or effective) constitutive equation for the multiphase piezoelectric composite in the form:

$$
\left[\begin{array}{c}
\overline{\mathbf{\sigma}} \\
\overline{\mathbf{D}}
\end{array}\right]=\left[\begin{array}{cc}
\mathbf{C}^{*} & \mathbf{e}^{* \mathrm{~T}} \\
\mathbf{e}^{*} & -\boldsymbol{\kappa}^{*}
\end{array}\right]\left[\begin{array}{c}
\overline{\boldsymbol{\varepsilon}} \\
-\overline{\mathbf{E}}
\end{array}\right]
$$

where $\mathbf{C}^{*}, \mathbf{e}^{*}$, and $\boldsymbol{\kappa}^{*}$ are the effective elastic, piezoelectric and dielectric matrices, respectively, given by:

$$
\left[\begin{array}{cc}
\mathbf{C}^{*} & \mathbf{e}^{* \mathrm{~T}} \\
\mathbf{e}^{*} & -\mathbf{K}^{*}
\end{array}\right]=\sum_{q=1}^{N_{q}} v^{(q)}\left[\begin{array}{cc}
\mathbf{C} & \mathbf{e}^{\mathrm{T}} \\
\mathbf{e} & -\mathbf{K}
\end{array}\right]^{(q)} \mathbf{A}^{(q)}
$$

\section{Numerical results}

\section{I. Homogenized responses}

In this section, the multiphysics FVDAM is employed to investigate the homogenized stiffness, piezoelectric and dielectric coefficients of multilayered piezoelectric composites. The effectiveness of the multiphysics FVDAM will be validated extensively, first against the theoretical values found in the literature, and then an in-house finite-element homogenization technique.

A flat multilayer can be considered as a special case of the wavy multilayers when the amplitude-towavelength ratio is zero. The multiphysics FVDAM is first validated against a theoretical solution based on the asymptotic homogenization model (AHM) developed by Kar-Gupta and Venkatesh (2013). Figure 2 illustrates the unit cell of a flat piezoelectric composite, consisting of two different phases, that is, the PVDF (white layer) and PZT-7A (gray layer). The elastic,

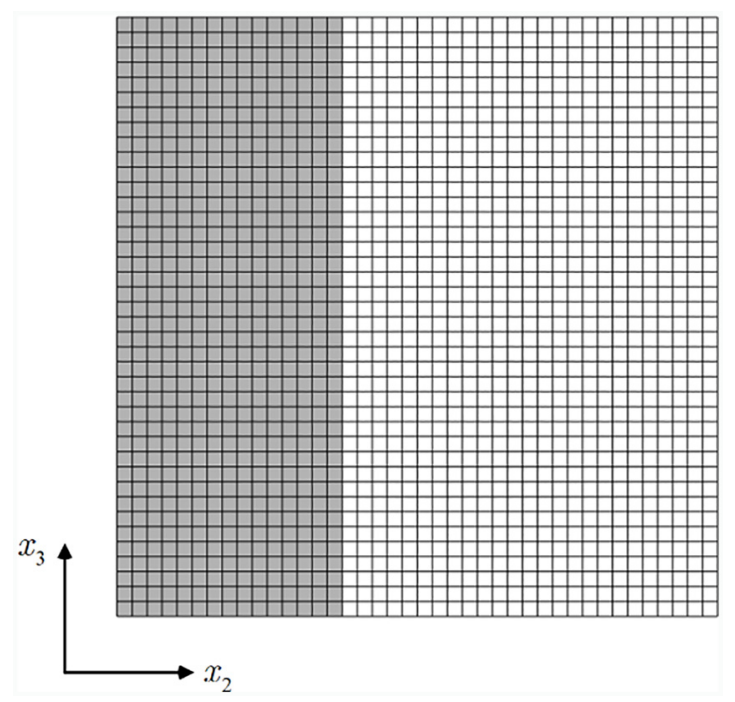

Figure 2. Unit cell of a bilayered piezoelectric composite used for comparison with the asymptotic homogenization model (Kar-Gupta and Venkatesh, 20I3). 
Table I. Electromechanical constants of constituent phases (Kar-Gupta and Venkatesh, 20I3).

\begin{tabular}{lccc}
\hline & PZT-7A & $\mathrm{BaTiO}_{3}$ & PVDF \\
\hline$C_{11}(\mathrm{GPa})$ & 131 & 145.5 & 4.84 \\
$C_{12}(\mathrm{GPa})$ & 74.2 & 65.94 & 2.22 \\
$C_{13}(\mathrm{GPa})$ & 74.2 & 65.94 & 2.22 \\
$C_{22}(\mathrm{GPa})$ & 148 & 150.4 & 4.84 \\
$C_{23}(\mathrm{GPa})$ & 76.2 & 65.63 & 2.72 \\
$C_{33}(\mathrm{GPa})$ & 148 & 150.4 & 4.84 \\
$C_{44}(\mathrm{GPa})$ & 35.9 & 42.37 & 1.06 \\
$C_{55}(\mathrm{GPa})$ & 25.3 & 43.86 & 5.26 \\
$\mathrm{C}_{66}(\mathrm{GPa})$ & 25.3 & 43.86 & 5.26 \\
$\mathrm{e}_{11}\left(\mathrm{Cm}{ }^{-2}\right)$ & 10.99 & 17.36 & -0.11 \\
$\mathrm{e}_{12}\left(\mathrm{Cm}{ }^{-2}\right)$ & -2.324 & -4.322 & 0.004 \\
$\mathrm{e}_{26}\left(\mathrm{Cm}{ }^{-2}\right)$ & 9.31 & 11.4 & -0.002 \\
$\kappa_{11}\left(\mathrm{nCV}^{-1} \mathrm{~m}^{-1}\right)$ & 2.081 & 15.1 & 0.07083 \\
$\kappa_{22}\left(\mathrm{nCV}^{-1} \mathrm{~m}^{-1}\right)$ & 3.984 & 12.8 & 0.06641 \\
\hline
\end{tabular}

piezoelectric, and dielectric coefficients which can be found in reference (Kar-Gupta and Venkatesh, 2013) are given in Table 1. The large property contrast between the constituent phases yields large stress and deformation gradients in the vicinity of the interface, providing a critical assessment of the multiphysics FVDAM. Figure 3 describes the comparison of the homogenized piezoelectric and dielectric coefficients generated by the multiphysics FVDAM and asymptotic homogenization model as a function of PZT-7A volume fraction. The results show an excellent level of accordance between the two approaches, providing a strong support for the multiphysics FVDAM approach.

We proceed to investigate the homogenized response of a multilayered composite consisting of PZT-7A and $\mathrm{BaTiO}_{3}$ piezoelectric phases of different volume fractions and poling directions. Figure 4 illustrates the geometric representation of a repeating unit cell of a sinusoidal shape multilayered composite. The gray layer is occupied by the PZT-7A while the two white layers are both occupied by $\mathrm{BaTiO}_{3}$. The volume fraction of the PZT-7A phase is denoted as $v_{f}$. Both of them are transversely isotropic with the axis of symmetry oriented in the $x_{1}$ direction, perpendicular to $x_{2}-x_{3}$ plane. According to the relatively poling direction of each ply, the piezoelectric multilayered composites can be either "transverse" or "longitudinal." In this work, the longitudinally poled multilayers are defined when all plies are poled along the $x_{1}$ direction, whereas the transversely poled multilayers are defined when the PZT-7A ply is poled in the $x_{1}$ direction while the $\mathrm{BaTiO}^{3}$ plies are poled in the $x_{2}$ direction.

Figure 5 presents the variation of selected homogenized elastic moduli of the PZT-7A/ $\mathrm{BaTiO}_{3}$ multilayered composite system as a function of PZT-7A volume content over a wide range of volume fractions for three different amplitude-to-wavelength ratios, namely, $0 \%$ (flat), $10 \%$, and $20 \%$. It should be noted that the poling directions of PZT-7A and $\mathrm{BaTiO}_{3}$ phases are both in $x_{1}$ direction. For validation purposes, the finite-element predictions are enclosed in the figure for comparison. We note that the imposition of the periodic boundary conditions is a difficult task for multilayered wavy architectures with curved boundaries in the commercial finite-element tools. Therefore, an in-house finite-element method (FEM) developed by the present authors (shown in the Appendix) is employed to generate reference results for comparison with the multiphysics FVDAM predictions. The inhouse multiphysics finite-element method mimics the unit cell solution methodology adopted by the multiphysics FVDAM theory. Hence the two sets of results may be compared on an equal footing. It is important to mention that both multiphysics FVDAM and inhouse FEM results are generated using MATLAB codes. The displacements, electric potentials, local stress, and electric fields are calculated directly without interpolation across element or subvolume interfaces. This is in contrast to the commercial finite-element codes that typically use averaging techniques in calculating local responses.

As expected, the homogenized moduli $C_{22}^{*}, C_{33}^{*}, C_{44}^{*}$, $C_{55}^{*}$, and $C_{66}^{*}$ decrease with increasing PZT-7A volume fractions while $C_{23}^{*}$ exhibits an opposite trend. This is due to the fact that the material stiffness coefficients of PZT-7A phase are smaller than those of $\mathrm{BaTiO}_{3}$ phase, except for $C_{23}^{*}$. The ply waviness has a pronounced effect on the homogenized moduli $C_{33}^{*}, C_{55}^{*}$, and $C_{66}^{*}$. $C_{33}^{*}$ and $C_{66}^{*}$ decrease with the increase of amplitude-towavelength ratio for a given PZT-7A volume fraction. In contrast, $C_{55}^{*}$ increases with the increase of amplitude-to-wavelength ratio for a given volume fraction. Increasing the PZT-7A volume fraction first accentuates the differences due to the ply waviness. After reaching a maximum just below the volume fraction of 0.5 , the ply waviness effect becomes less remarkable with increasing PZT-7A volume fraction. The multiphysics FVDAM predictions coincide with the finite-element results in the entire volume fraction range and for all the amplitude-to-wavelength ratios, providing good support for the developed FVDAM technique.

Comparisons of homogenized piezoelectric and dielectric coefficients generated by the multiphysics FVDAM and finite-element approaches as a function of PZT-volume fraction for three different ply amplitude-to-wavelength ratios are provided in Figures 6 and 7, respectively. Similar to the previous observations, no visible differences are observed between the finite-element and finite-volume predictions over a wide range of volume fractions. While the ply waviness has a negligible effect on the axial piezoelectric and dielectric coefficients, namely, $e_{11}^{*}, e_{12}^{*}, e_{13}^{*}$, and $\kappa_{11}^{*}$, it plays an important role in affecting the transverse coefficients, namely, $e_{26}^{*}, e_{35}^{*}, \kappa_{22}^{*}$, and $\kappa_{33}^{*}$. 

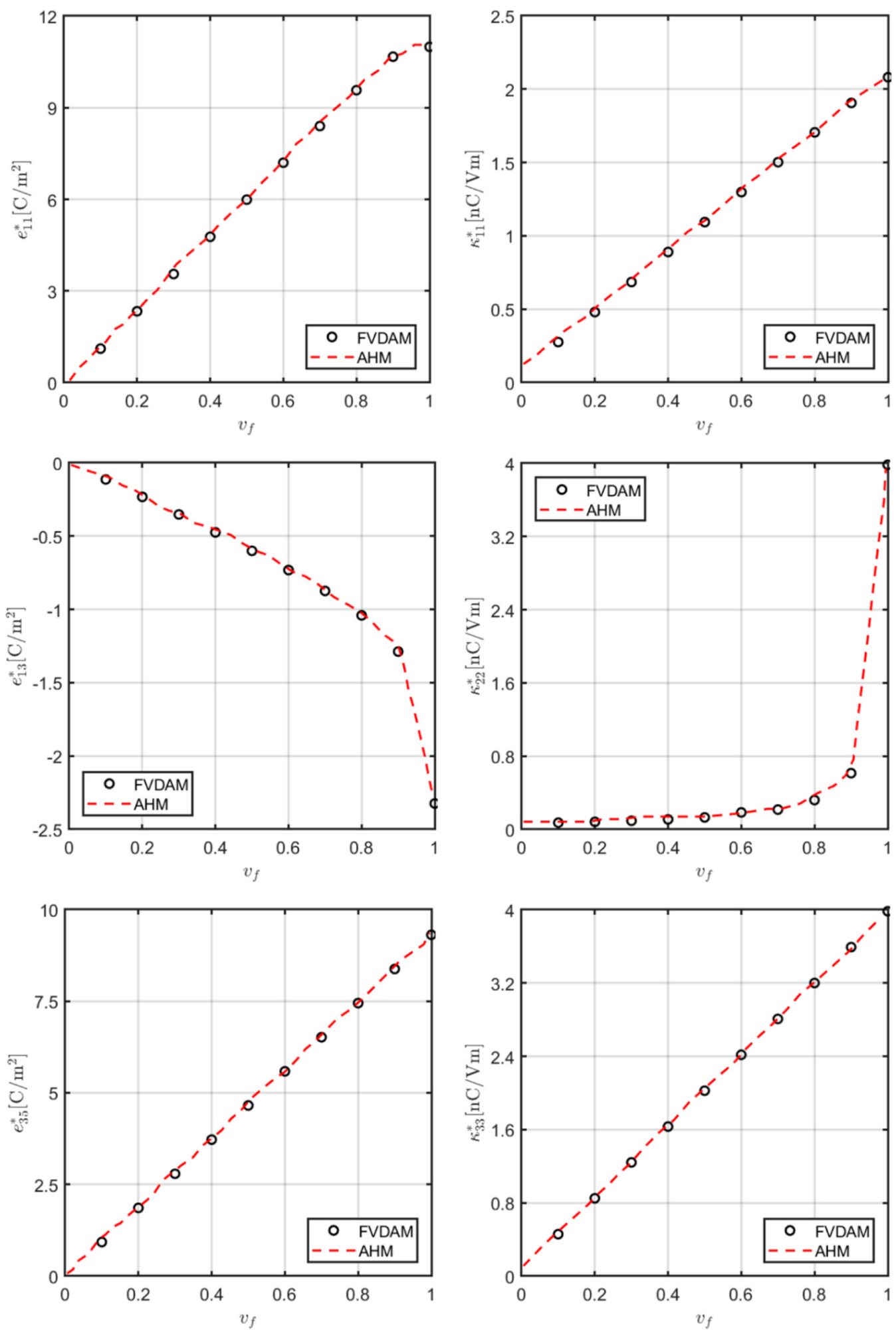

Figure 3. Comparison of selected homogenized piezoelectric and dielectric coefficients as a function of PZT-7A volume fraction generated by the multiphysics FVDAM and asymptotic homogenization model (Kar-Gupta and Venkatesh, 20I3).

In order to demonstrate the effect of the poling direction on the homogenized piezoelectric and dielectric coefficients of the piezoelectric multilayered composites, a transverse $\mathrm{PZT}-7 \mathrm{~A} / \mathrm{BaTiO}_{3}$ multilayered composite is considered. Table 2 presents a detailed comparison of the complete set of piezoelectric and dielectric coefficients of $40 \%$ PZT-7A/BaTiO 3 multilayered composites with different poling directions. The 


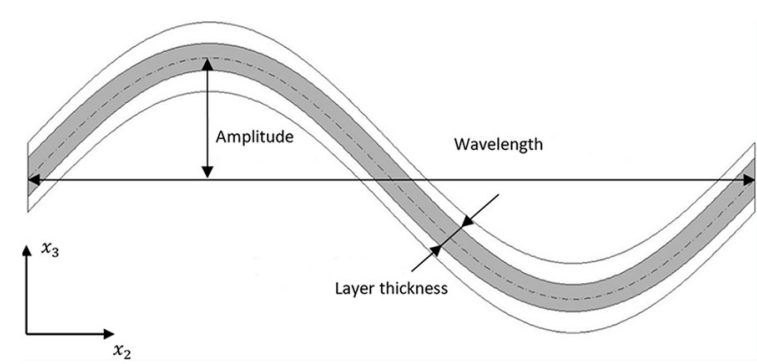

Figure 4. Geometric representation of a repeating unit cell representative of multilayered composites with wavy architectures.

ply amplitude-to-wavelength ratios are prescribed as $10 \%$. It is noted that different results may be obtained by changing the relative poling direction of the PZT$7 \mathrm{~A}$ and $\mathrm{BaTiO}_{3}$ plies. The transverse isotropy ceases to hold in the case of transversely poled composites. The most interesting behavior is the moduli $e_{16}^{*}, e_{21}^{*}, e_{22}^{*}, e_{23}^{*}$, $e_{34}^{*}$ and $\kappa_{12}^{*}, \kappa_{21}^{*}$ that are present in the transversely poled composites but not in either of the individual phase or the longitudinal composites. Therefore, those coefficients are new material properties that are exhibited by composites with designated poling directions.

Besides the above-mentioned new material properties that do not exist in the longitudinal material system, certain homogenized piezoelectric properties of the transversely poled multilayered $\mathrm{PZT}-7 \mathrm{~A} / \mathrm{BaTiO}^{3}$ composites vary much more significantly as the change of volume fraction of PZT-7A and amplitude-towavelength ratio. For instance, in the flat longitudinally poled composites as shown in Figure $6, e_{26}^{*}$ varies within the range of [9.7-11.4] C/m $\mathrm{m}^{2}$ and the difference between lower bound and higher bound is $1.7 \mathrm{C} / \mathrm{m}^{2}$, while in the flat transversely poled multilayers as shown in Figure 8, $e_{26}^{*}$ varies within the range of [0-8.125] C/ $\mathrm{m}^{2}$ with a much larger difference between the lower bound and higher bound, $8.125 \mathrm{C} / \mathrm{m}^{2}$. Meanwhile, in the longitudinally poled multilayers, the most significant change of $e_{26}^{*}$ due to amplitude-to-wavelength ratio variation is $0.2 \mathrm{C} / \mathrm{m}^{2}$, occurring at the volume fraction of 0.35 . In contrast, in the transversely poled composites, the most significant change of $e_{26}^{*}$ due to the amplitude-to-wavelength ratio is much larger, that is $1.25 \mathrm{C} / \mathrm{m}^{2}$, occurring at the volume fraction of 0.5 . $e_{35}^{*}$ also has a similar trend. In the flat longitudinally poled multilayers, $e_{35}^{*}$ varies in the range of [9.811.5] $\mathrm{C} / \mathrm{m}^{2}$ with $1.7 \mathrm{C} / \mathrm{m}^{2}$ difference between the lower and upper bound. In contrast, for the flat transversely poled multilayers, $e_{35}^{*}$ varies in the range of $[0-9.3] \mathrm{C} /$ $\mathrm{m}^{2}$ with 7.5 times higher difference. Meanwhile, in the longitudinally poled multilayers, the highest difference of $e_{35}^{*}$ due to amplitude-to-wavelength ratio variation is $0.18 \mathrm{C} / \mathrm{m}^{2}$, occurring at volume fraction of 0.25 . In contrast, in the transversely poled multilayers, the highest difference of $e_{35}^{*}$ due to amplitude-towavelength ratio variation is nine times higher, occurring at volume fraction of 0.45 . As we can see, in transversely poled multilayers, the volume fraction and amplitude-to-wavelength ratio have a significant effect on the homogenized piezoelectric properties, $e_{26}^{*}, e_{35}^{*}$ and offer a large design space to tailor the homogenized piezoelectric properties.

Figures 7 and 9 show the variations of selected homogenized dielectric properties of longitudinally and transversely poled multilayers at different volume fractions for three different amplitude-to-wavelength ratios: $0 \%, 10 \%$, and $20 \%$. Dielectric properties, $k_{22}^{*}$ and $k_{33}^{*}$ of longitudinally poled multilayers are more sensitive to the change of amplitude-to-wavelength ratio than the transversely poled multilayers. However, the biggest difference between the longitudinally and transversely poled multilayers is the introduction of a coupling dielectric property, $k_{12}^{*}$, which reaches a maximal value at the volume fraction of 0.38 . In particular, the nonlinearity of $k_{12}^{*}$ increases significantly as the increase of amplitude-to-wavelength ratios. The new findings open new opportunities to design new applications of piezoelectric devices.

Table 3 shows comparison of the selected homogenized properties of transversely-poled PZT-7A/BaTiO 3 multilayered composites generated by the multiphysics FVDAM and FEM approaches at two different amplitude-to-wavelength ratios, that is, $10 \%$ and $20 \%$. The relative errors defined by: $\mid\left((.)_{\text {FVDAM }}-\right.$ $\left.(.)_{\mathrm{FEM}}\right) /(.)_{\mathrm{FEM}} \mid \times 100 \%$ are also included in Table 3 . The PZT-7A phase volume fraction is fixed at $40 \%$. As observed, the multiphysics FVDAM and FEM agree to two or three significant digits for all cases. The relative percentage errors of the multiphysics FVDAM predictions relative to the FEM results are negligible. The above comparison provides additional evidence of the accuracy of the multiphysics FVDAM for simulating the multilayered piezoelectric architectures. It should be mentioned that the finite-element homogenized properties are generated based on the same mesh discretization as the multiphysics FVDAM technique, namely, $120 \times 40$. We have verified that for the finiteelement homogenization, this mesh refinement produces results with sufficient accuracy, hence the finiteelement homogenized properties are reliable as reference solutions.

\subsection{Local stress/electric fields}

The ability to correctly recover the full-field localized stress and electric displacement distributions within the composite microstructures helps to understand the underlying deformation mechanisms of the composite materials and structures. To the best knowledge of the present authors, there have been very few reliable tools that yield accurate localized field distribution within 

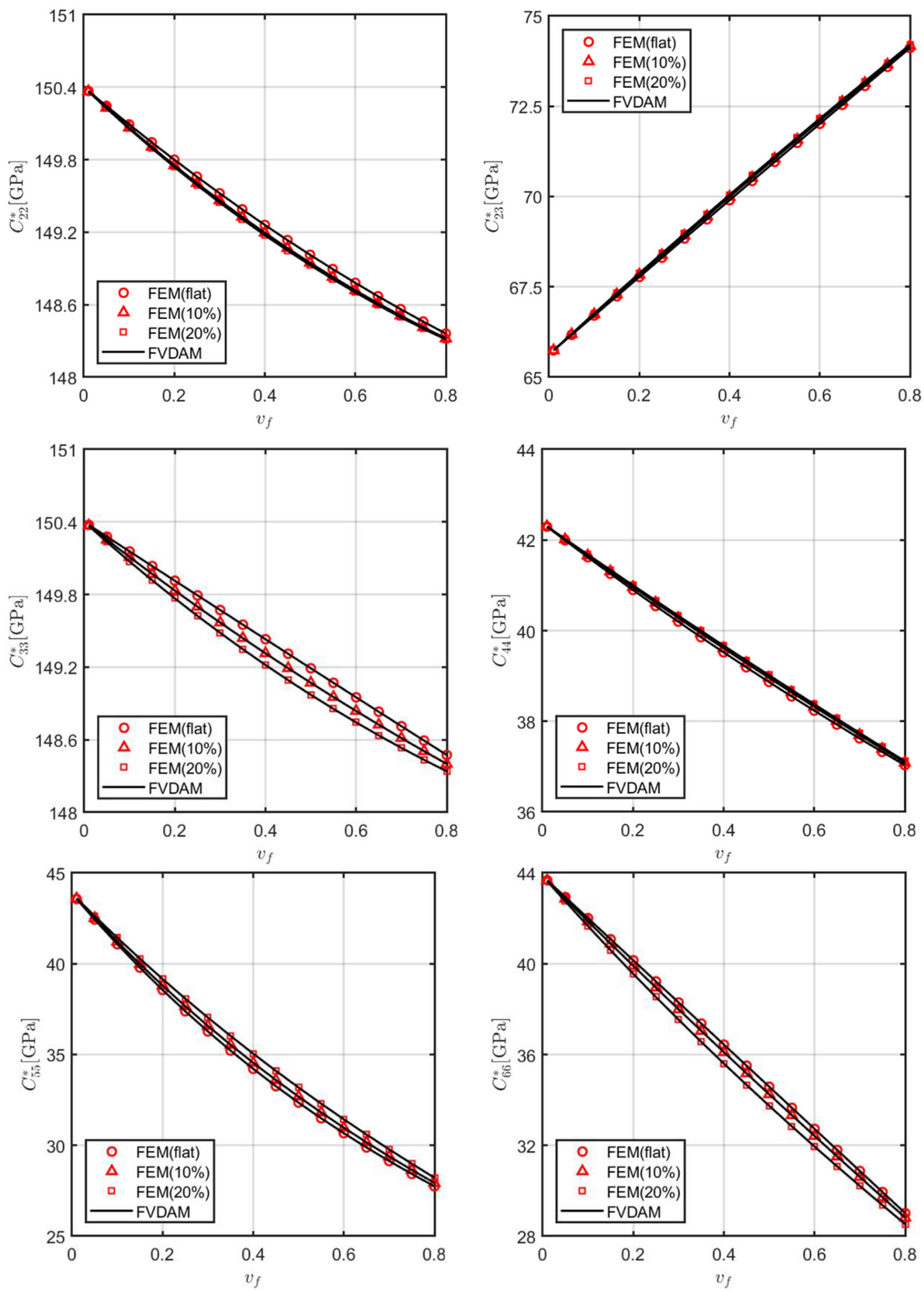

Figure 5. Comparison of homogenized mechanical properties of multilayered PZT-7A/BaTiO ${ }_{3}$ architectures as a function of PZT7A volume faction for three different amplitude-to-wavelength ratios: $0 \%$ (flat), $10 \%$ and $20 \%$.

the heterogeneous microstructures with only two exceptions, namely, the finite-element and finite-volume techniques that explicitly impose periodic boundary conditions. cf. Chen et al. (2018a) and Chen and Wang $(2018,2020)$. Figure 10 presents a comparison of the local electric displacements $D_{2}$ and $D_{3}$ distributions with the imposition of unit homogenized electric displacement $\bar{D}_{2}$ generated by the multiphysics FVDAM and an in-house finite-element code for the $40 \%$ PZT-7A $/ \mathrm{BaTiO}_{3}$ composites with an amplitudeto-wavelength ratio of $20 \%$. Both the multiphysics FVDAM and finite-element are generated using the same mesh discretization, namely, $120 \times 40$. It is observed that the concentration of electric 

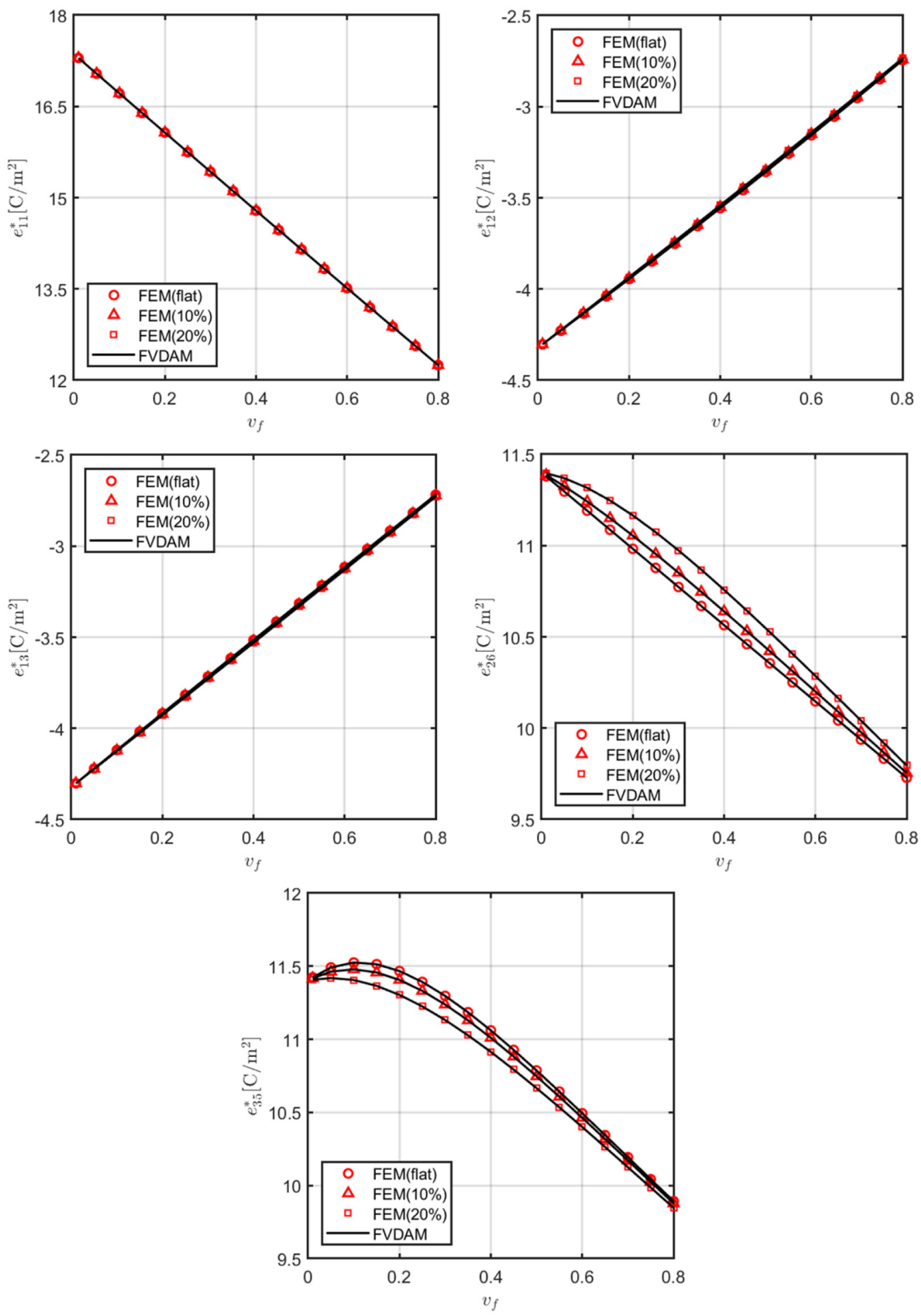

Figure 6. Comparison of selected homogenized piezoelectric properties of multilayered PZT-7A/BaTiO ${ }_{3}$ architectures as a function of PZT-7A volume faction for three different amplitude-to-wavelength ratios: $0 \%$ (flat), $10 \%$, and $20 \%$.

displacements which are nonuniform within the wavy microstructures is significant. The correlation between the two computational approaches is remarkable, providing good support for the developed multiphysics FVDAM technique.

Figure 11 illustrates the comparison of the local axial shear stress $\sigma_{12}$ and $\sigma_{13}$ distributions during the unidirectional electric displacement loading by $\bar{D}_{2}=1 \mathrm{C} / \mathrm{m}^{2}$, reflecting the coupling effect between the mechanical response and applied electric field. Once again, the differences between the multiphysics FVDAM and finite element predictions are small.

The effect of the ply amplitude-to-wavelength ratio on the localized stress and electric displacement 

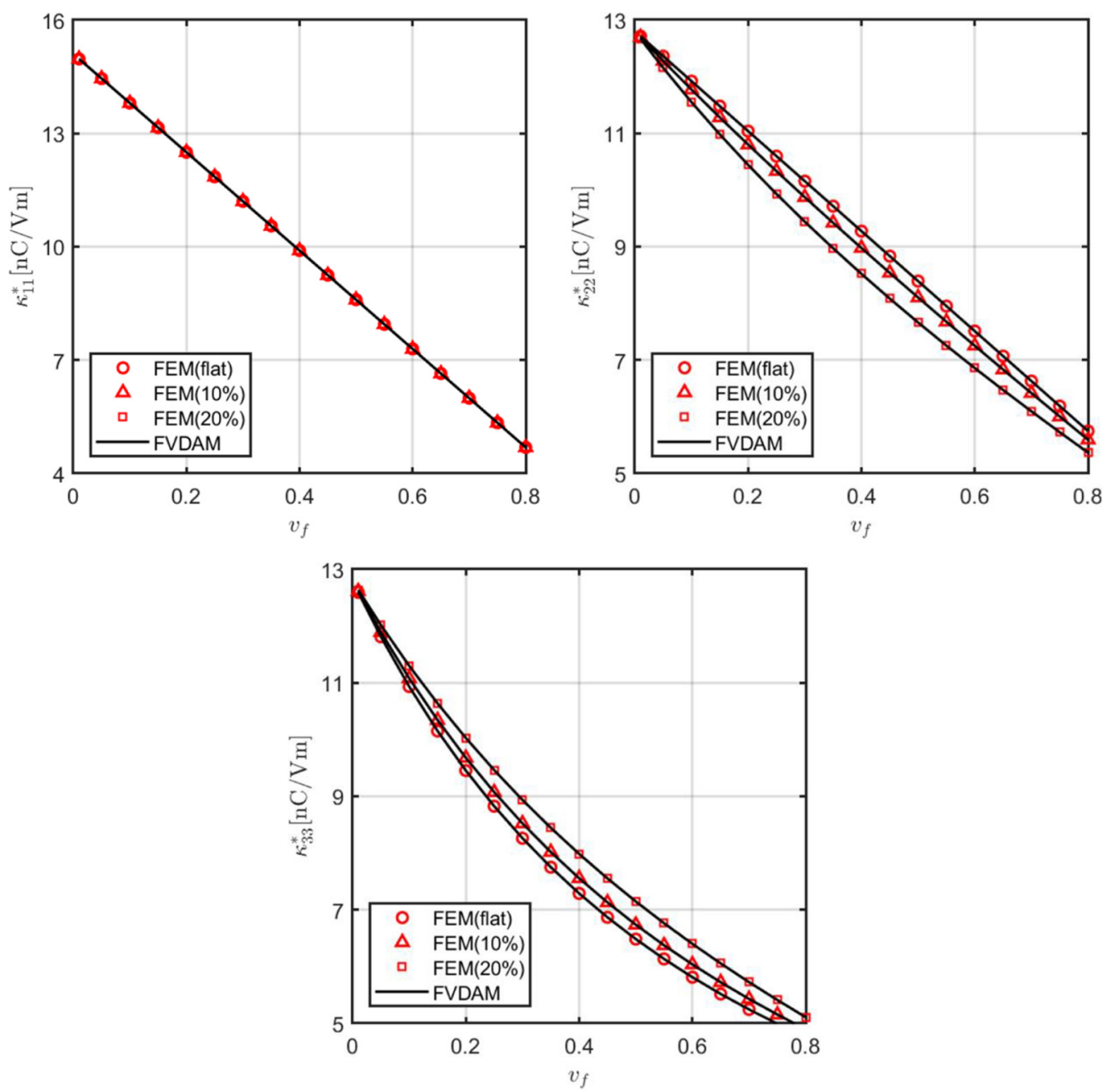

Figure 7. Comparison of selected homogenized dielectric properties of multilayered PZT-7A/BaTiO ${ }_{3}$ architectures as a function of PZT-7A volume faction for three different amplitude-to-wavelength ratios: $0 \%$ (flat), $10 \%$, and $20 \%$.

Table 2. Comparison of homogenized piezoelectric and dielectric properties of multilayered architectures with different poling directions.

\begin{tabular}{lllllll}
\hline & Piezoelectric coefficients & & Dielectric coefficients \\
\hline Longitudinally Poled & {$\left[\begin{array}{lllllll}14.79 & -3.55 & -3.52 & 0 & 0 & 0 \\
0 & 0 & 0 & 0 & 0 & 10.64 \\
0 & 0 & 0 & 0 & 11.01 & 0\end{array}\right]$} & {$\left[\begin{array}{ccc}9.90 & 0 & 0 \\
0 & 8.98 & 0 \\
0 & 7.55\end{array}\right]$} \\
Transversely Poled & {$\left[\begin{array}{llllll}4.46 & -1.03 & -0.91 & 0 & 0 & 6.71 \\
-2.44 & 9.50 & -2.25 & 0 & 0 & 4.44 \\
0 & 0 & 0 & 4.56 & 7.13 & 0\end{array}\right]$} & {$\left[\begin{array}{ccc}8.65 & -0.21 & 0 \\
-0.21 & 10.62 & 0 \\
0 & 8.84\end{array}\right]$} \\
\hline
\end{tabular}

distributions is illustrated in Figure 12. We consider a unidirectional multilayered composite comprising $40 \%$ PZT-7A for three different amplitude-to-wavelength ratios. The composites are subjected to unidirectional electric displacement loading $\bar{D}_{2}=1 \mathrm{C} / \mathrm{m}^{2}$. In the case of a flat multilayer, the shear stress $\sigma_{12}$ and electric displacement $D_{2}$ are uniform within each phase, whereas the shear stress $\sigma_{13}$ and electric displacement $D_{3}$ are zero, indicating no coupling in $\sigma_{13}$ and $D_{3}$. In contrast, in the case of a wavy multilayer, the shear stress $\sigma_{13}$ and electric displacement $D_{3}$ arise and the local stress and electric displacement become nonuniform under the same loading condition. As the ply amplitude-towavelength ratio increases, larger stress and electric field concentration are observed between the interface of different plies. Hence the location and conditions for 

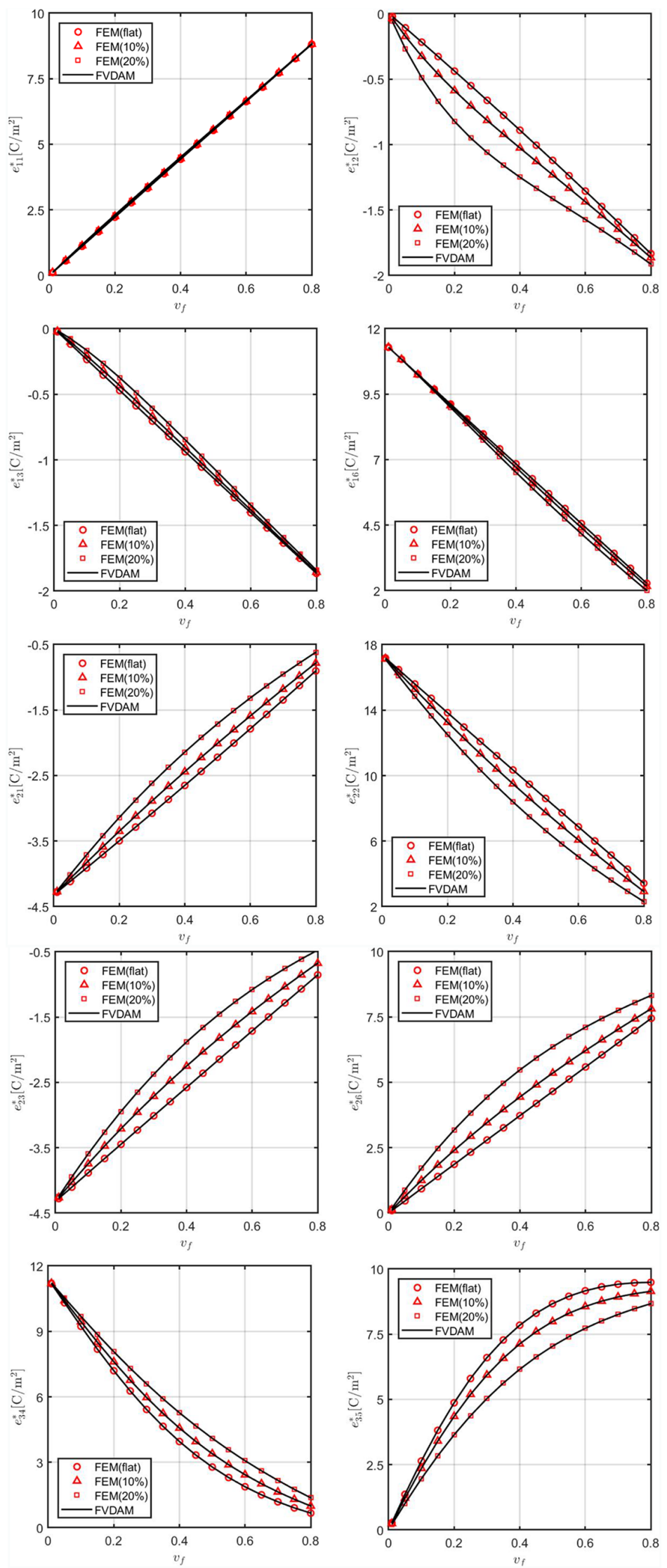

Figure 8. Comparison of homogenized piezoelectric properties of transversely poled multilayered $\mathrm{PZT}-7 \mathrm{~A} / \mathrm{BaTiO} \mathrm{architectures}^{3}$ as a function of PZT-7A volume faction for three different amplitude-to-wavelength ratios: $0 \%$ (flat), $10 \%$, and $20 \%$. 

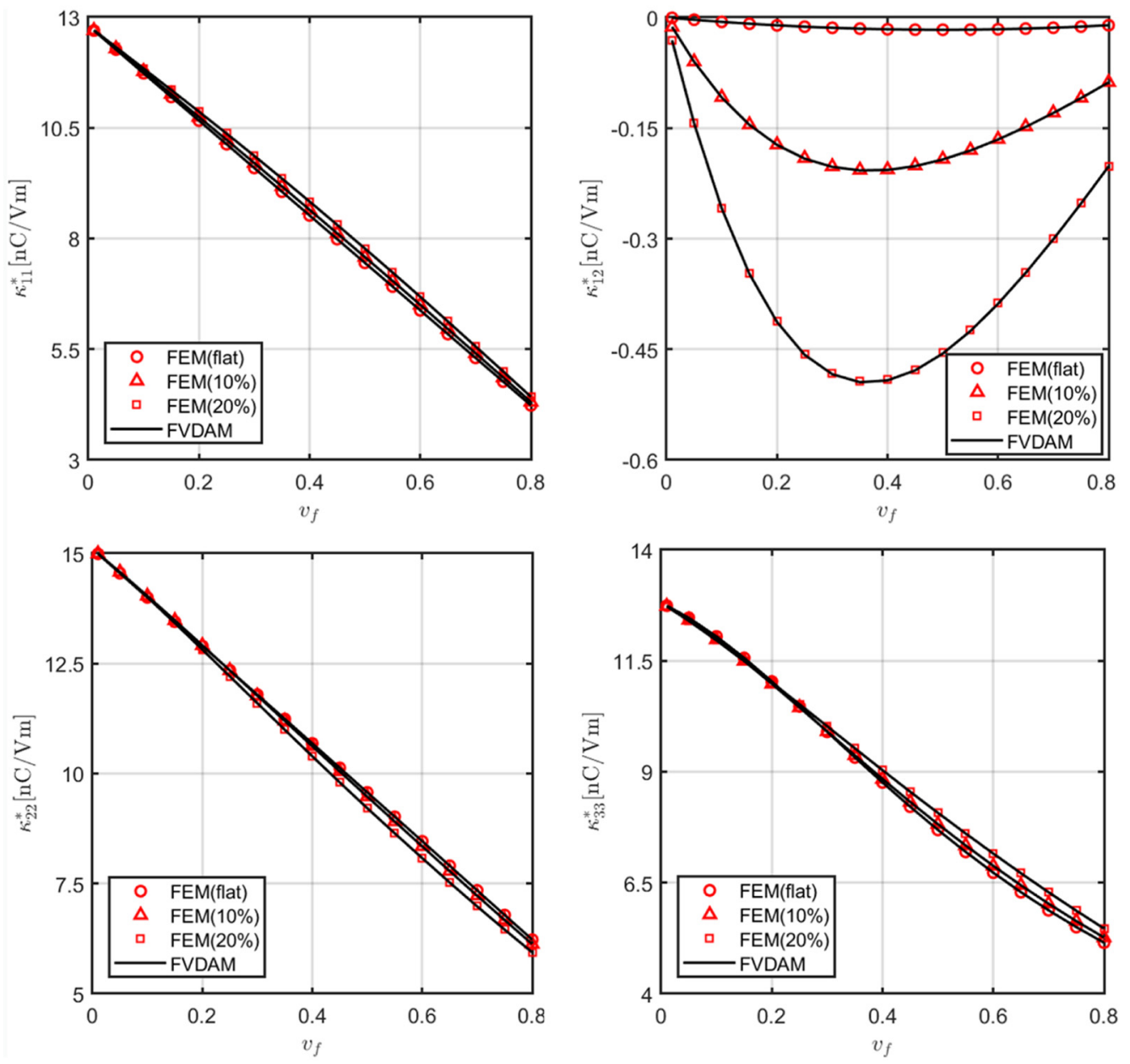

Figure 9. Comparison of homogenized dielectric properties of transversely poled multilayered $\mathrm{PZT}-7 \mathrm{~A} / \mathrm{BaTiO} \mathrm{O}_{3}$ architectures as a function of PZT-7A volume faction.

Table 3. Quantitative comparison of the homogenized properties of a transversely-poled $\mathrm{PZT}-7 \mathrm{~A} / \mathrm{BaTiO}_{3}$ multilayered composite with $40 \%$ PZT-7A volume content for two different amplitude-to-wavelength ratios.

\begin{tabular}{|c|c|c|c|c|c|c|c|c|}
\hline & $\begin{array}{l}\mathrm{C}_{22}^{*} \\
(\mathrm{GPa})\end{array}$ & $\begin{array}{l}\mathrm{C}_{44}^{*} \\
(\mathrm{GPa})\end{array}$ & $\begin{array}{l}\mathrm{e}_{12}^{*} \\
\left(\mathrm{C} / \mathrm{m}^{2}\right)\end{array}$ & $\begin{array}{l}\mathrm{e}_{22}^{*} \\
\left(\mathrm{C} / \mathrm{m}^{2}\right)\end{array}$ & $\begin{array}{l}\mathrm{e}_{35}^{*} \\
\left(\mathrm{C} / \mathrm{m}^{2}\right)\end{array}$ & $\begin{array}{l}\kappa_{11}^{*} \\
(\mathrm{nC} / \mathrm{Vm})\end{array}$ & $\begin{array}{l}\kappa_{12}^{*} \\
(\mathrm{nC} / \mathrm{Vm})\end{array}$ & $\begin{array}{l}\kappa_{33}^{*} \\
(\mathrm{nC} / \mathrm{Vm})\end{array}$ \\
\hline \multicolumn{9}{|c|}{ Wavelength-to-amplitude ratio: $10 \%$} \\
\hline FVDAM & 150.2174 & 42.2780 & -1.0262 & 9.4946 & 7.1288 & 8.6476 & -0.2070 & 8.8370 \\
\hline FEM & 150.2172 & 42.2785 & $-1.026 \mid$ & 9.4949 & 7.1287 & 8.6476 & -0.2069 & 8.8369 \\
\hline Relative Error (\%) & 0.0001 & 0.001 & 0.010 & 0.003 & 0.001 & 0 & 0.048 & 0.001 \\
\hline \multicolumn{9}{|c|}{ Wavelength-to-amplitude ratio: $20 \%$} \\
\hline FVDAM & $151.8 \mid 53$ & 41.646 & -1.2495 & 8.3956 & 6.1538 & 8.8346 & -0.4923 & 9.0349 \\
\hline FEM & 151.8155 & 41.648 & -1.2497 & 8.3960 & 6.1537 & 8.8344 & -0.4923 & 9.0356 \\
\hline Relative Error (\%) & 0 & 0.005 & 0.016 & 0.005 & 0.024 & 0.002 & 0 & 0.008 \\
\hline
\end{tabular}

the onset of mechanical failure in the piezoelectric composites could be predicted.

\section{Assessment of accuracy}

Multiphysics FVDAM and FEM are fundamentally different in the way of satisfying equilibrium and conservation equations in the discretized domain of the unit cell. In FVDAM, equilibrium and conservation equations are directly satisfied in an integral sense for every subvolume, hence the automatic satisfaction of global equilibrium and conservation. In contrast, in FEM, equilibrium and conservation equations are achieved via the minimization of total potential energy, which requires sufficient mesh refinement to achieve 

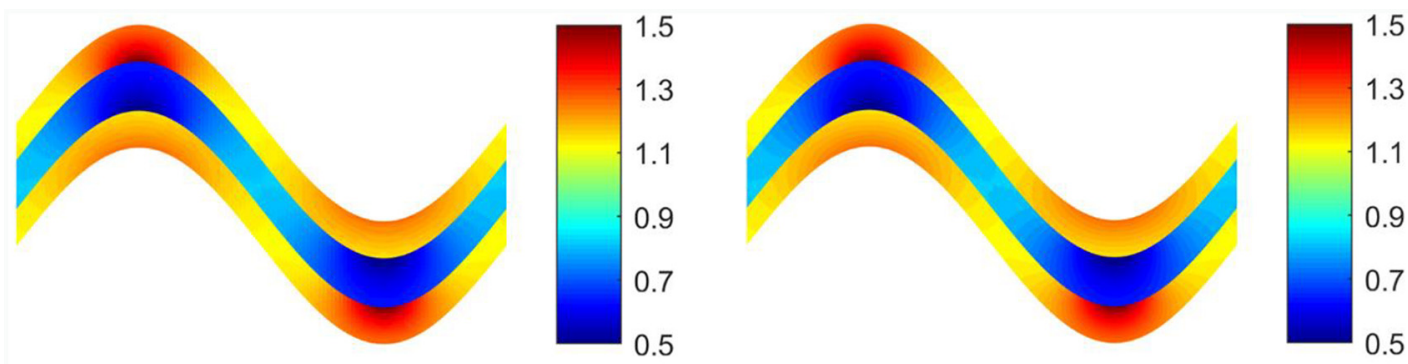

(a)
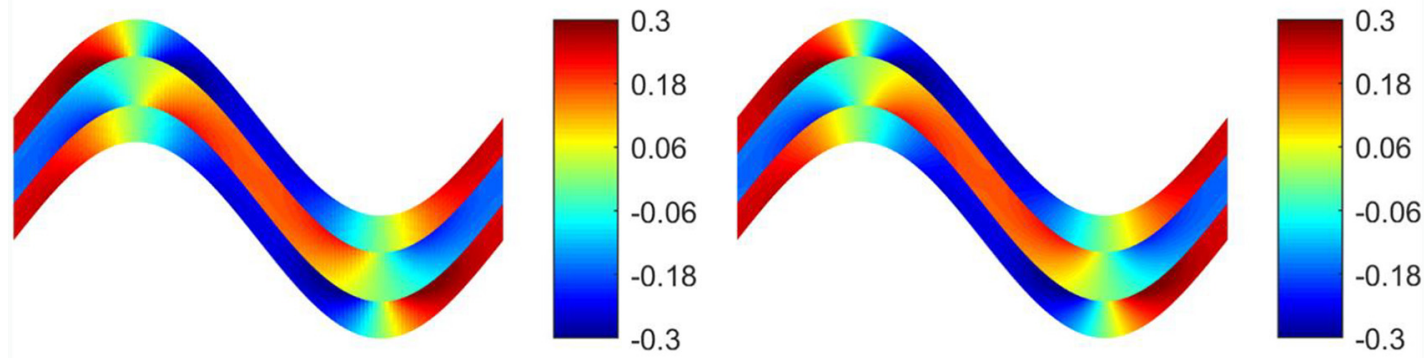

(b)

Figure 10. Comparison of local electric displacement distributions of longitudinally poled multilayered $\mathrm{PZT}-7 \mathrm{~A} / \mathrm{BaTiO} \mathrm{T}_{3}$ architectures with 40\% PZT-7A volume content during unidirectional electric displacement loading by $\bar{D}_{2}=1 \mathrm{IC} / \mathrm{m}^{2}$ generated by the FVDAM (left column) and FEM (right column): (a) $D_{2}$ and (b) $D_{3}$.
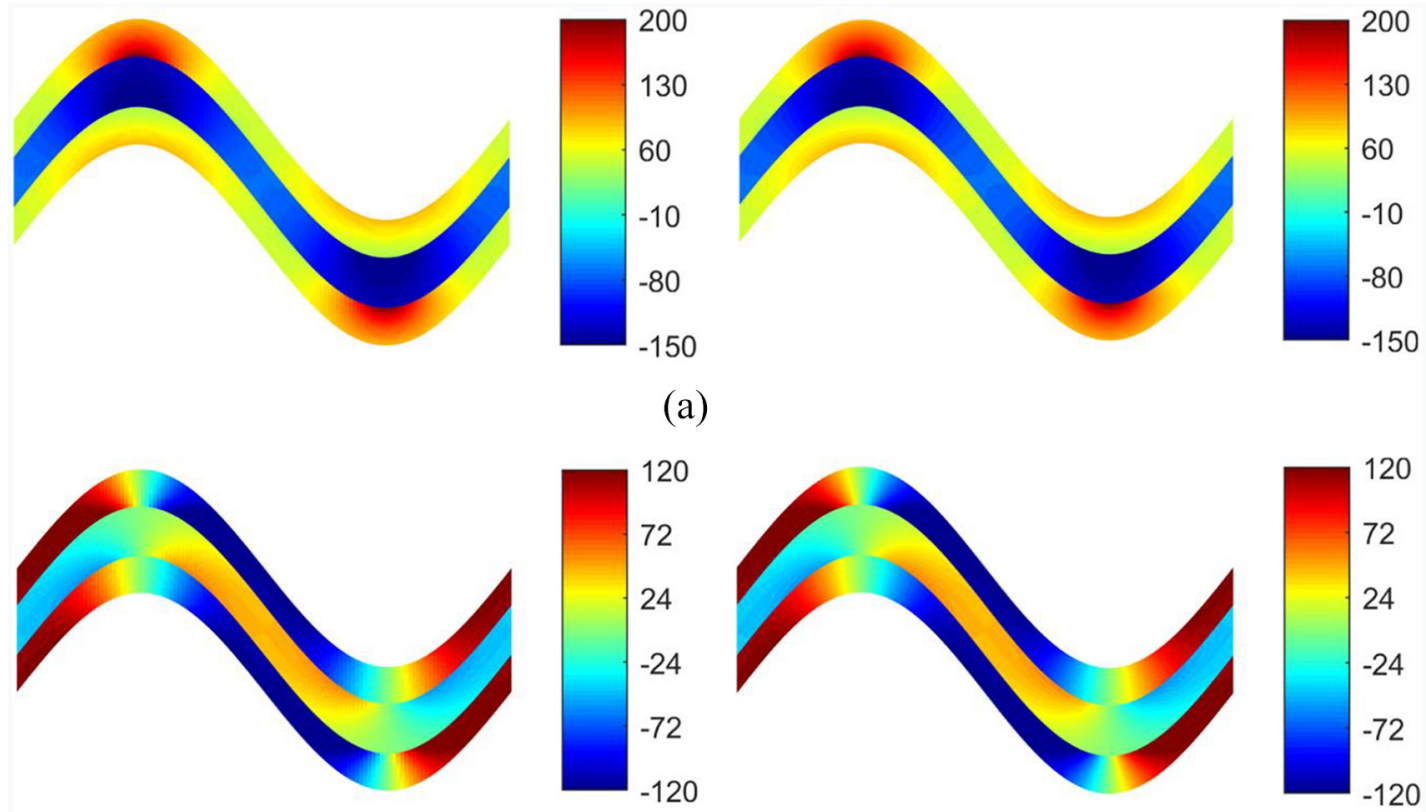

(b)

Figure I I. Comparison of local stress distributions of longitudinally poled multilayered $\mathrm{PZT}-7 \mathrm{~A} / \mathrm{BaTiO}_{3}$ architectures with $40 \%$ PZT-7A volume content during unidirectional electric displacement loading by $\bar{D}_{2}=1 \mathrm{C} / \mathrm{m}^{2}$ generated by the FVDAM (left column) and FEM (right column): (a) $\sigma_{12}$ and (b) $\sigma_{13}$.

global equilibrium and conservation, and then equilibrium and conservation at the element level. To gauge the satisfaction of equilibrium and conservation of an element or a subvolume for current piezoelectric analysis, unbalanced average stress and unbalanced average electric displacement can be defined via the Average Stress Theorem and the Average Electric Displacement Theorem. 

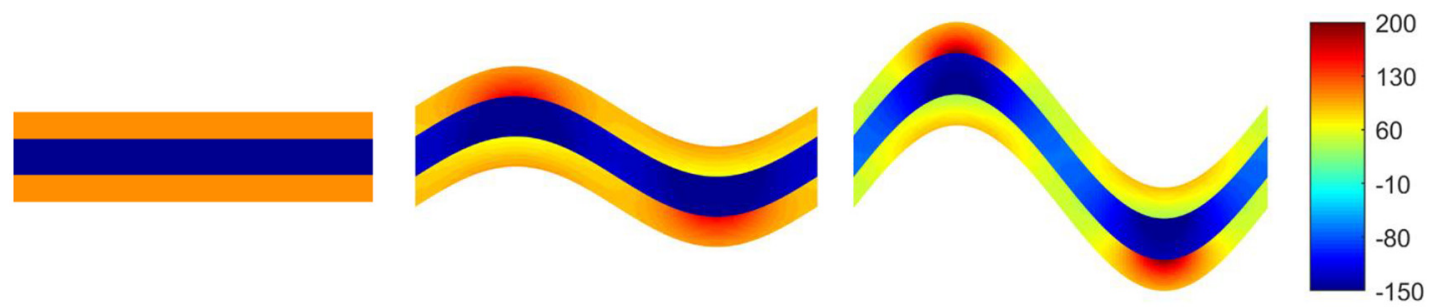

(a)
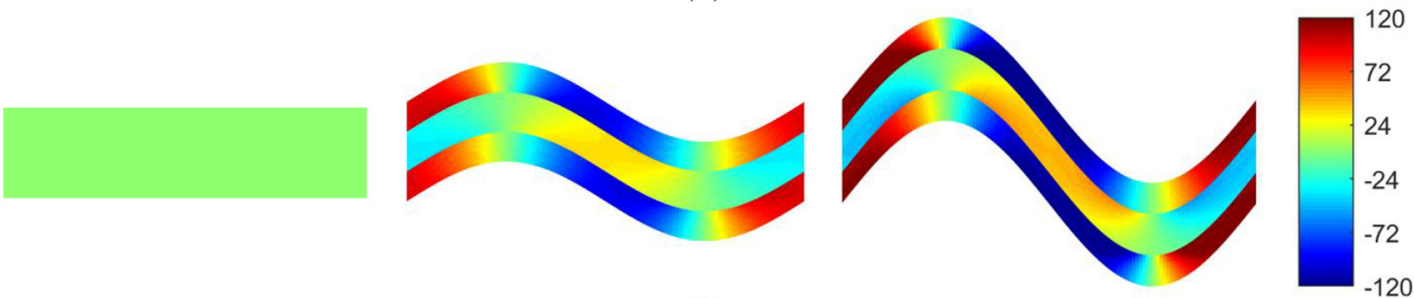

(b)
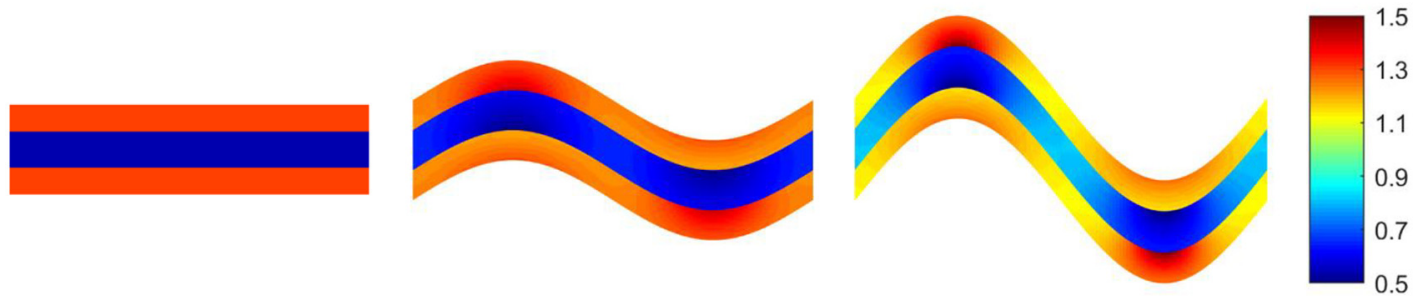

(c)
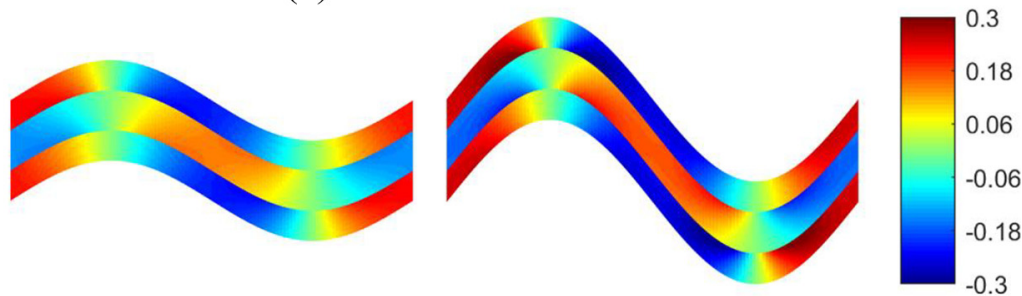

(d)

Figure I2. Effect of amplitude-to-wavelength ratio on the local stress and electric displacement distributions of longitudinally poled multilayered PZT-7A/BaTiO 3 architectures with $40 \%$ PZT-7A volume content during unidirectional electric displacement loading by $\bar{D}_{2}=1 \mathrm{C} / \mathrm{m}^{2}$ : flat (left column); $10 \%$ (middle column); $20 \%$ (right column): (a) $\sigma_{12}$ and (b) $\sigma_{13}$ (c) $D_{2}$, and (d) $D_{3}$.

According to the Average Stress Theorem, for an arbitrary domain in equilibrium status, the average stress $\hat{\sigma}_{i j}$ can be calculated by integrating the surface tractions as follows:

$$
\hat{\sigma}_{i j}=\frac{1}{V} \oiint_{S} T_{i} x_{j} d S=\frac{1}{V} \oiint_{S} \sigma_{i k} n_{k} x_{j} d S
$$

Converting the surface integral to volume integral using Gauss's Theorem, we obtain

$$
\begin{aligned}
\hat{\sigma}_{i j} & =\frac{1}{V} \oiint_{S} \sigma_{i k} n_{k} x_{j} d S=\frac{1}{V} \iiint_{V} \frac{\partial}{\partial x_{k}}\left(\sigma_{i k} x_{j}\right) d V \\
& =\frac{1}{V} \iiint_{V}\left[\frac{\partial \sigma_{i k}}{\partial x_{k}} x_{j}+\sigma_{i j}\right] d V
\end{aligned}
$$

In the absence of body forces, when equilibrium is satisfied in a pointwise sense, $\frac{\partial \sigma_{i k}}{\partial x_{k}}=0$, the above integral reduces to the standard definition for the volume-averaged stress, as follows:

$$
\frac{1}{V} \iiint_{V}\left[\frac{\partial \sigma_{i k}}{\partial x_{k}} x_{j}+\frac{\partial x_{j}}{\partial x_{k}} \sigma_{i k}\right] \mathrm{d} V=\frac{1}{V} \iiint_{V} \sigma_{i j} \mathrm{~d} V=\bar{\sigma}_{i j}
$$

It should be noted that in FEM the equilibrium at the subdomain is not automatically satisfied unless the mesh discretization is sufficiently refined. Therefore, we can define unbalanced average stress to gauge the numerical convergence as follows:

$$
\Delta \bar{\sigma}_{i j}=\bar{\sigma}_{i j}-\hat{\sigma}_{i j}=\frac{1}{V} \iiint_{V} \sigma_{i j} \mathrm{~d} V-\frac{1}{V} \oiint_{S} T_{i} x_{j} \mathrm{~d} S
$$

For each element or subvolume, the unbalanced average stress can be expressed as: 


$$
\Delta \bar{\sigma}_{i j}^{(q)}=\frac{1}{V_{q}} \iiint_{V_{q}} \sigma_{i j} \mathrm{~d} V-\frac{1}{V} \oiint_{S_{q}} T_{i} x_{j} \mathrm{~d} S
$$

While for the entire unit cell, a global unbalanced average stress can be calculated by summing up all the absolute value of unbalanced average stress for each element or subvolume as follows:

$$
\Delta \bar{\sigma}_{i j}=\sum_{q=1}^{N_{q}} \frac{V_{q}}{V}\left|\Delta \bar{\sigma}_{i j}^{(q)}\right|
$$

Similarly, the unbalanced average electric displacement can be defined and utilized to gauge the convergence of the electric field. According to the Average Electric Displacement Theorem, for an arbitrary domain in the absence of electric charge, the average electric displacement $\hat{D}_{i}$ can be calculated by integrating electric displacements along the domain surface as follows:

$$
\hat{D}_{i}=\frac{1}{V} \oiint_{S} D_{i} n_{i} x_{j} \mathrm{~d} S
$$

Converting the surface integral to volume integral using Gauss's Theorem, we obtain

$$
\hat{D}_{i}=\frac{1}{V} \oiint_{S}\left(D_{i} x_{j}\right) n_{i} \mathrm{~d} S=\frac{1}{V} \iiint_{V}\left[\frac{\partial D_{i}}{\partial x_{i}} x_{j}+D_{i}\right] \mathrm{d} V
$$

In the absence of electric charge, when the conservation equation is satisfied in a pointwise sense, $\iiint_{V} \frac{\partial D_{i}}{\partial x_{i}}=0$, the above integral reduces to the standard definition for the volume-averaged electric displacement, as follows:

$$
\frac{1}{V} \iiint_{V}\left[\frac{\partial D_{i}}{\partial x_{i}} x_{j}+D_{i}\right] \mathrm{d} V=\frac{1}{V} \iiint_{V} D_{i} \mathrm{~d} V=\bar{D}_{i}
$$

In FEM the conservation at the subdomain is not automatically satisfied unless the mesh discretization is sufficiently refined. Therefore, we can define an unbalanced average electric displacement to gauge the numerical convergence as follows:

$$
\Delta \bar{D}_{i}=\bar{D}_{i}-\hat{D}_{i}=\frac{1}{V} \iiint_{V} D_{i} \mathrm{~d} V-\frac{1}{V} \oiint_{S} D_{i} n_{i} x_{j} \mathrm{~d} S
$$

For each subvolume/element, the unbalanced electric displacement can be expressed as:

$$
\Delta \bar{D}_{i}=\bar{D}_{i}-\hat{D}_{i}^{(q)}=\frac{1}{V_{q}} \iiint_{V_{q}} D_{i} \mathrm{~d} V-\frac{1}{V_{q}} \oiint_{S_{q}} D_{i} n_{i} x_{j} \mathrm{~d} S
$$

While for the entire unit cell, a global unbalanced average electric displacement can be calculated by summing up all the absolute value of unbalanced average electric displacement for each element or subvolume as follows:

$$
\Delta \bar{D}_{i}=\sum_{q=1}^{N_{q}} \frac{V_{q}}{V}\left|\Delta D_{i}^{(q)}\right|
$$

To illustrate the degree of equilibrium and local conservation is satisfied as a function of mesh refinement in the FEM and FVDAM approaches, the piezoelectric multilayers are analyzed under electric displacement loading $\bar{D}_{2}$ of $1 \mathrm{C} / \mathrm{m}^{2}$. Figure 13 illustrates the variation of overall unbalanced shear stresses $\Delta \bar{\sigma}_{13}, \Delta \bar{\sigma}_{12}$ and normalized unbalanced electric displacements $\Delta \bar{D}_{2} / \bar{D}_{2}, \Delta \bar{D}_{3} / \bar{D}_{2}$ over a wide range of mesh refinement from $12 \times 4$ to $120 \times 40$ and it shows the unbalanced quantity decreases with the increase of element number in FEM. Particularly the magnitude of $\Delta \bar{\sigma}_{13}, \Delta \bar{\sigma}_{12}$ decreases from the value of $40 \mathrm{MPa}$ and $63 \mathrm{MPa}$ to the value of $4 \mathrm{MPa}$ and $6 \mathrm{MPa}$ with the coarsest $12 \times 4$ and the finest mesh $120 \times 40$ discretizations, respectively. And the percentage of $\Delta \bar{D}_{2} / \bar{D}_{2}$ and $\Delta \bar{D}_{3} / \bar{D}_{2}$ changes from the value of $18.6 \%$ and $12 \%$ to the value of $1.71 \%$ and $1.14 \%$, respectively. It is important for researchers and engineers to notice that a mesh refinement study needs to be conducted toward the analysis of Multiphysics Piezoelectric Composites if the FEMbased Multiphysics micromechanics homogenization technique is utilized. In contrast, in multiphysics FVDAM, both the unbalanced average stress and unbalanced average electric displacement remain zero and they do not change as the mesh refinement. This is because in the FVDAM framework the equilibrium and local conservation equations are enforced during the process of analytical evaluation of the subvolume's generalized local stiffness matrices (Chen et al. 2018a).

Figures 14 and 15 present the distributions of normalized unbalanced average stress $\Delta \hat{\sigma}_{13}$ and electric displacement $\Delta \hat{D}_{2}$ at element/subvolume level for three types of mesh refinement, $30 \times 8,60 \times 14$, and $120 \times 40$, in FEM and FVDAM. And $\Delta \hat{\sigma}_{13}$ is normalized by the maximal stress, $\sigma_{13}^{\max }$ in the analysis domain and $\Delta \hat{D}_{2}$ is normalized by the applied electric displacement, $\bar{D}_{2}$. In FVDAM, the unbalanced average stress and electric displacement for the entire domain are exactly zeros due to the exact satisfaction of the equilibrium equation and conservation equation at the subvolume level. In contrast, in FEM, the value of normalized unbalanced average stress and electric displacement varies significantly, in the range of $[0 \%-$ $30 \%$ ]. The unbalanced average stress and electric displacement are more widespread in the unit cell with coarser mesh than the unit cell with refined mesh. It is interesting to observe the unbalanced average stress and electric displacement concentrates along the interface of two layers. This is consistent with the fact that local stress and electric displacement concentration 

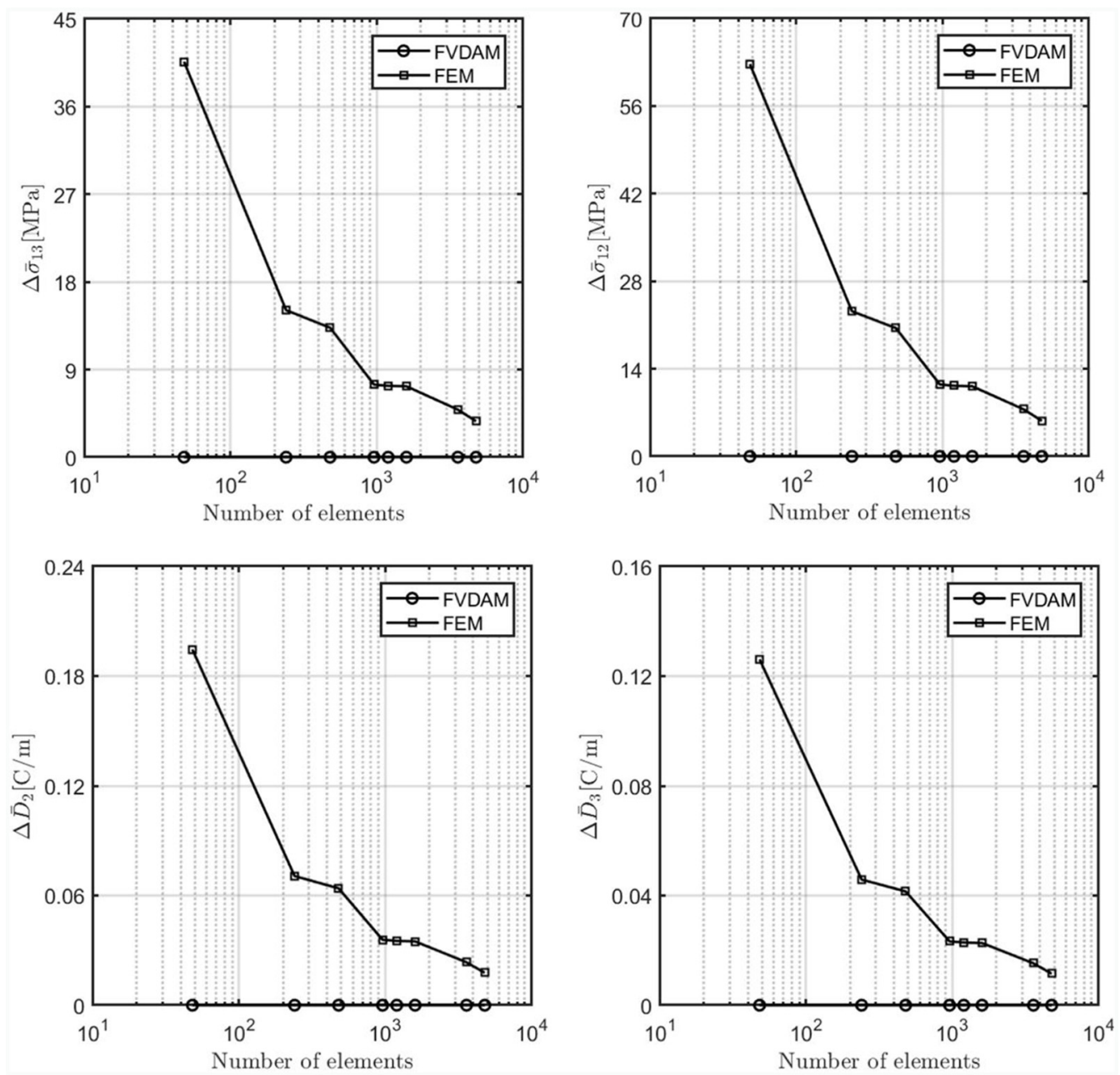

Figure 13. Comparison of unbalanced stresses and non-conservation of electric displacements as a function of mesh refinement generated by the FVDAM and FEM approaches.

occurs along the interface of different constituents with distinctive material properties, and equilibrium and conservation are more difficult to be satisfied in the FEM framework due to the large gradient of stress and electric displacement fields. Therefore, the multiphysics FVDAM can serve as a more reliable tool to predict the onset of failure due to large mechanical stress in the piezoelectric composites without the concern of mesh refinements.

\section{Discussions and future work}

The increasing applications of the piezoelectric multilayered composites in emerging technologies require an accurate yet efficient micromechanics model to study their homogenized and localized responses such that laborious experimental test can be avoided or reduced. Relative to the numerical technique such as the finiteelement method, the strengths of the present multiphysics FVDAM are several folds. First, the FVDAM satisfies the local (hence global) governing differential equations (stress equilibrium equations and conservation equation) in each volume at each level of mesh refinement. The finite-element method, however, is based on the minimization of the global potential energy of the analysis domain. Therefore, sufficient mesh refinement may be required to ensure local equilibrium and conservation conditions. Secondly, continuity conditions are applied on both interfacial tractions and displacements, as well as electric displacements and potentials, across all common subvolume interfaces, whereas only continuity of displacements and electric potentials are enforced at the adjacent nodes in the finite-element techniques. In the case of multiphased and heterogeneous materials, the satisfaction of continuity of both tractions and displacements, as well as electric displacements and potentials, is both demanding and essential, especially along the interface separating different phases. Thirdly, as demonstrated recently by the present authors, cf. Tu and Chen (2020), the FVDAM is naturally well-suited for simulating the damage initiation and evolution in composite materials 


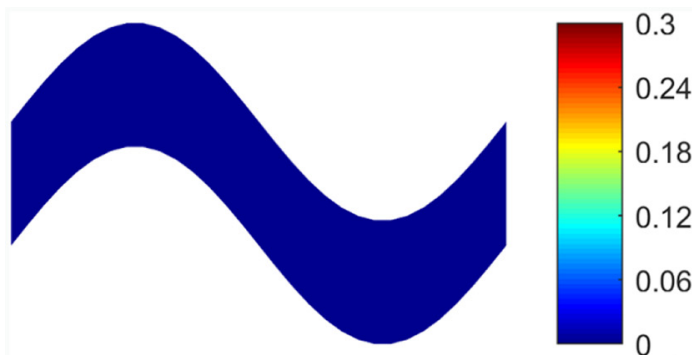

(a)

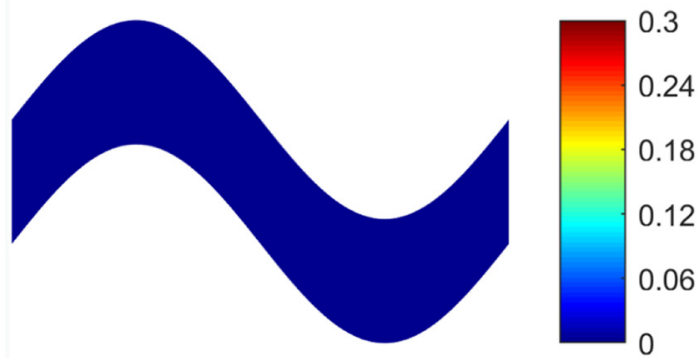

(b)
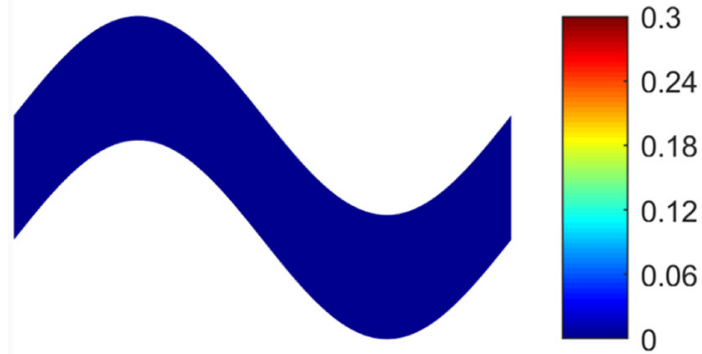

(c)
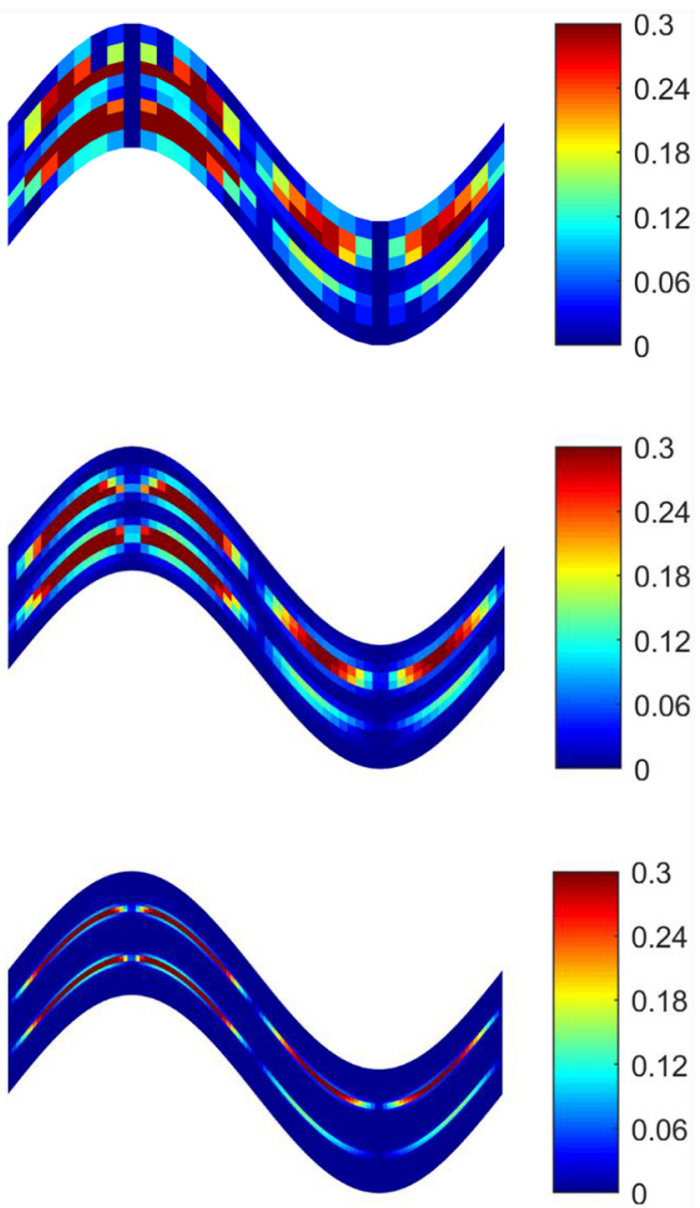

Figure 14. Comparison of subvolume unbalanced stress $\Delta \hat{\sigma}_{13} / \sigma_{13}^{\max }(\mathbf{y})$ as a function of mesh refinement generated by the FVDAM (left column) and FEM (right column) approaches: (a) $30 \times 8$, (b) $60 \times 14$, and (c) $120 \times 40$.

without experiencing the convergence and numerical issues common to the finite-element approaches. The ability to simulate evolutionary damage sets the FVDAM apart from other micromechanics models, as damage simulation used to largely rely on the finiteelement techniques. The present work provides a foundation for the future extension for simulating damage initiation and evolution in piezoelectric composites.

Further, the usability of the FVDAM is at least twofold. The FVDAM can be easily used in a standalone manner in the parametric studies aimed at understanding the microstructure-homogenized structure relationship, as conducted in the present work. Secondly, to make full use of the potential offered by the proposed method, the FVDAM can be incorporated into a structure analysis code for multiscale applications. For instance, the structure version of the finite-volume theory is incorporated into topology optimization code for compliance minimization of continuum linear elastic structures, cf. Araujo et al. (2020). The multiphysics FVDAM with some modification is also suitable for incorporation into a structural analysis tool, such as Abaqus via the user material subroutine. This work is in progress and will be reported elsewhere in our future publications.

\section{Summary and conclusion}

With the aid of multiphysics FVDAM and in-house micromechanical multiphysics finite-element model, a comprehensive study is conducted to understand the effects of poling direction, volume fraction, and amplitude-to-wavelength ratio on the homogenized and localized response of wavy multilayered piezoelectric BaTiO3/PZT-7A architectures. Both approaches generated highly agreeable results, while multiphysics FVDAM proves a better convergence in satisfying the conservation equations. The principal conclusions of this study are as follows:

1. In the longitudinally poled multilayers, the increase of PZT-7A volume fraction first accentuates the variation of homogenized mechanical properties of longitudinally poled multilayers due to the amplitude-to-wavelength ratio, while 

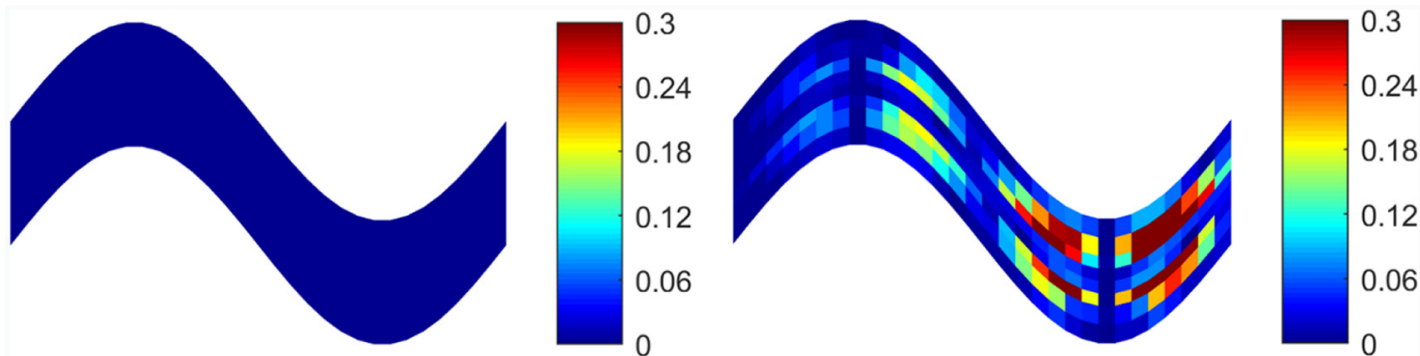

(a)
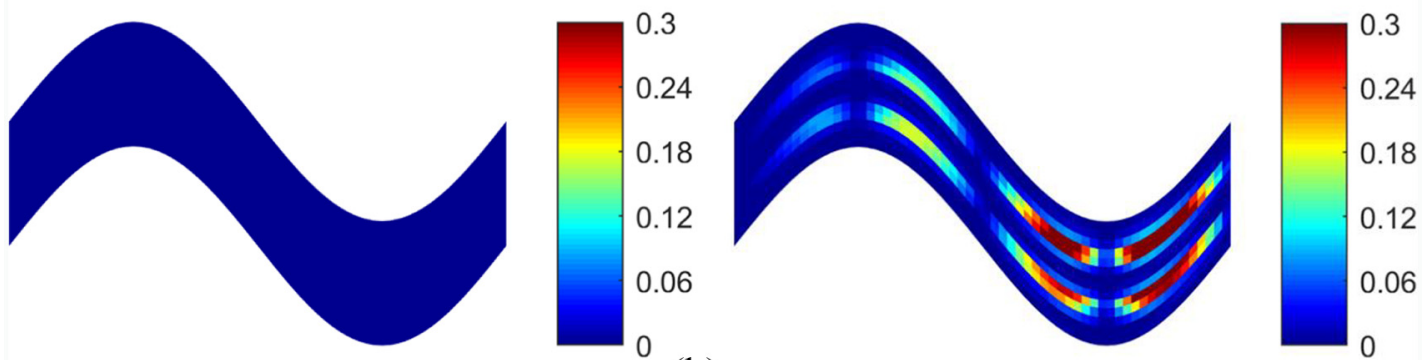

(b)
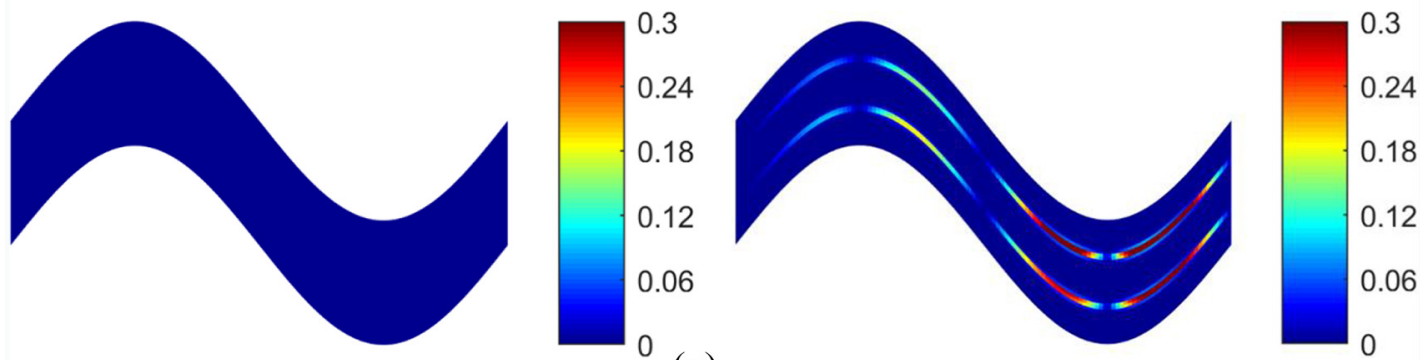

(c)

Figure 15. Comparison of subvolume non-conservation of electric displacements $\Delta \hat{D}_{2} / \bar{D}_{2}$ as a function of mesh refinement generated by the FVDAM (left column) and FEM (right column) approaches: (a) $30 \times 8$, (b) $60 \times 14$, and (c) $120 \times 40$.

the ply waviness effects decrease once the volume fraction is above $50 \%$.

2. Comparing with the longitudinally poled multilayers, the transversely poled multilayered composites produce six new homogenized piezoelectric properties, $e_{16}^{*}, e_{21}^{*}, e_{22}^{*}, e_{23}^{*}, e_{34}^{*}$ and two new homogenized dielectric properties, $\boldsymbol{\kappa}_{12}^{*}$, $\kappa_{21}^{*}$. Meanwhile, for the transversely poled multilayers, the amplitude-to-wavelength ratio has a significant effect on the homogenized piezoelectric properties, $e_{26}^{*}, e_{35}^{*}$ and the dielectric properties, $\boldsymbol{\kappa}_{12}^{*}, \boldsymbol{\kappa}_{21}^{*}$, and offers a higher degree of tunability for the material system.

3. The concentration of stress and electric displacement field typically occurs at the interface of PZT-7A/BaTiO ${ }^{3}$ layers. As the increase of amplitude-to-wavelength ratio, the stress and electric displacement concentration also increase. And the multiphysics FVDAM and FEM approaches enable us to predict the onset of mechanical failure.

4. FEM-based Multiphysics mechanics is more sensitive to mesh refinement. Multiphysics
FVDAM exhibits a better convergence due to its direct satisfaction of the conservation equation at the subvolume level in an integral sense.

\section{Declaration of conflicting interests}

The author(s) declared no potential conflicts of interest with respect to the research, authorship, and/or publication of this article.

\section{Funding}

The author(s) disclosed receipt of the following financial support for the research, authorship, and/or publication of this article: The first author W. Tu acknowledges the support of Jiangsu University Research Startup Fund for Senior Talent.

\section{ORCID iD}

Qiang Chen (iD https://orcid.org/0000-0002-1623-8756

\section{References}

Araujo MVO, Lages EN and Cavalcante MAA (2020) Checkerboard free topology optimization for compliance minimization applying the finite-volume theory. Mechanics Research Communications 108: 103581. 
Asano S, Nishimura S, Ikeda Y, et al. (2020) Energy harvester for safety shoes using parallel piezoelectric links. Sensors and Actuators A: Physical 309:112000.

Camarena E and Yu W (2019) Improved analytical homogenization of the piezoelectric macro-fiber composite: Active layer embedded among passive layers. Smart Materials and Structures 28(4): 045021.

Cannata JM, Williams JA, Zhou Q, et al. (2006) Development of a 35-MHz piezo-composite ultrasound array for medical imaging. IEEE Transactions on Ultrasonics, Ferroelectrics, and Frequency Control 53(1): 224-236.

Cavalcante, MA and Pindera, MJ (2016) Generalized FVDAM theory for elastic -plastic periodic materials. International Journal of Plasticity 77: 90-117.

Cavalcante MA, Pindera MJ and Khatam H (2012) Finitevolume micromechanics of periodic materials: Past, present and future. Composites Part B: Engineering 43(6): 2521-2543.

Challagulla KS and Venkatesh TA (2009) Electromechanical response of 2-2 layered piezoelectric composites: A micromechanical model based on the asymptotic homogenization method. Philosophical Magazine 89(14): 1197-1222.

Charalambakis N (2010) Homogenization techniques and micromechanics. A survey and perspectives. Applied Mechanics Reviews 63(3): 030803.

Chatzigeorgiou G, Benaarbia A and Meraghni F (2019) Piezoelectric-piezomagnetic behaviour of coated long fiber composites accounting for eigenfields. Mechanics of Materials 138: 103157.

Chen Q and Pindera MJ (2020) Homogenization and localization of elastic-plastic nanoporous materials with GurtinMurdoch interfaces: An assessment of computational approaches. International Journal of Plasticity 124: 42-70.

Chen Q, Tu W, Liu R, et al. (2018a) Parametric multiphysics finite-volume theory for periodic composites with thermoelectro-elastic phases. Journal of Intelligent Material Systems and Structures 29(4): 530-552.

Chen Q and Wang G (2018) Homogenized and localized responses of coated magnetostrictive porous materials and structures. Composite Structures 187: 102-115.

Chen Q and Wang G (2020) Computationally-efficient homogenization and localization of unidirectional piezoelectric composites with partially cracked interface. Composite Structures 232: 111452.

Chen Q, Wang G and Pindera MJ (2018b) Homogenization and localization of nanoporous composites-A critical review and new developments. Composites Part B: Engineering 155: 329-368.

Dubey MK and Panda S (2019) Shear actuation mechanism and shear-based actuation capability of an obliquely reinforced piezoelectric fibre composite in active control of annular plates. Journal of Intelligent Material Systems and Structures 30(16): 2447-2463.

Feng GH and Huang ZW (2013) A smart acoustic emission and mechanical impedance hybrid sensor with static force detecting and dynamic measuring capabilities. In: 2013 transducers \& eurosensors XXVII: The 17th international conference on solid-state sensors, actuators and microsystems (TRANSDUCERS \& EUROSENSORS XXVII), Barcelona, Spain, 16-20 June, pp.936-939. New York: IEEE.
Feng GH, Tsai MY and Chen JS (2009) Development of structure enhanced micromachined acoustic emission sensors with wide-bandwidth and improved sensitivity. In: SENSORS, 2009 IEEE, Christchurch, New Zealand, 2528 October, pp. 463-466. New York: IEEE.

Feng X, Yang BD, Liu Y, et al. (2011) Stretchable ferroelectric nanoribbons with wavy configurations on elastomeric substrates. Acs Nano 5(4): 3326-3332.

Han Y, Cao Y, Zhao J, et al. (2016) A self-powered insole for human motion recognition. Sensors 16(9): 1502.

Hill R (1963) Elastic properties of reinforced solids: Some theoretical principles. Journal of the Mechanics and Physics of Solids 11(5): 357-372.

Jiang CP and Cheung YK (2001) An exact solution for the three-phase piezoelectric cylinder model under antiplane shear and its applications to piezoelectric composites. International Journal of Solids and Structures 38(28-29): 4777-4796.

Kar-Gupta R, Marcheselli C and Venkatesh TA (2008) Electromechanical response of 1-3 piezoelectric composites: Effect of fiber shape. Journal of Applied Physics 104(2): 024105.

Kar-Gupta R and Venkatesh TA (2005) Electromechanical response of 1-3 piezoelectric composites: Effect of poling characteristics. Journal of Applied Physics 98(5): 054102.

Kar-Gupta R and Venkatesh TA (2007) Electromechanical response of 1-3 piezoelectric composites: An analytical model. Acta Materialia 55(3): 1093-1108.

Kar-Gupta R and Venkatesh TA (2013) Electromechanical response of (2-2) layered piezoelectric composites. Smart Materials and Structures 22(2): 025035.

Katz A, Trinh C, Wright J, et al. (2015) Plastic strain localization in periodic materials with wavy brick-and-mortar architectures and its effect on the homogenized response. Composites Part B: Engineering 68: 270-280.

Khatam H and Pindera MJ (2009a) Parametric finite-volume micromechanics of periodic materials with elastoplastic phases. International Journal of Plasticity 25(7): 1386-1411.

Khatam H and Pindera MJ (2009b) Thermo-elastic moduli of periodic multilayers with wavy architectures. Composites Part B: Engineering 40(1): 50-64.

Kim BC, Baltazar A and Kim JY (2009) Effective properties of multi-layered multi-functional composites. Advanced Composite Materials 18(2): 153-166.

Kim BS, Ji JH, Kim HT, et al. (2020) Improved multilayered $(\mathrm{Bi}, \mathrm{Sc}) \mathrm{O} 3-(\mathrm{Pb}, \mathrm{Ti}) \mathrm{O} 3$ piezoelectric energy harvesters based on impedance matching technique. Sensors 20(7): 1958.

Li JY and Dunn ML (1998) Micromechanics of magnetoelectroelastic composite materials: Average fields and effective behavior. Journal of Intelligent Material Systems and Structures 9(6): 404-416.

Li J, Huang H and Morita T (2019) Stepping piezoelectric actuators with large working stroke for nano-positioning systems: A review. Sensors and Actuators A: Physical 292: 39-51.

Liu H, Zhong J, Lee C, et al. (2018) A comprehensive review on piezoelectric energy harvesting technology: Materials, mechanisms, and applications. Applied Physics Reviews 5(4): 041306. 
Na WS and Baek J (2018) A review of the piezoelectric electromechanical impedance based structural health monitoring technique for engineering structures. Sensors 18(5): 1307.

Panda S and Kumar Dubey M (2020) A balanced laminate of piezoelectric fiber composite for improved shear piezoelectric actuation of beams. Mechanics of Advanced Materials and Structures 27(15): 1291-1303.

Qi Y, Kim J, Nguyen TD, et al. (2011) Enhanced piezoelectricity and stretchability in energy harvesting devices fabricated from buckled PZT ribbons. Nano Letters 11(3): 1331-1336.

Singh S, Kumar Gupta V and Mukherjee S (2018) Piezoelectric Based Energy Harvester Embedded in Shoe for Wearable Electronics. Materials Physics \& Mechanics 37(2): 159-167.

Sun KH and Kim YY (2010) Layout design optimization for magneto-electro-elastic laminate composites for maximized energy conversion under mechanical loading. Smart Materials and Structures 19(5): 055008.

Tu W and Chen Q (2020) Homogenization and localization of unidirectional fiber-reinforced composites with evolving damage by FVDAM and FEM approaches: A critical assessment. Engineering Fracture Mechanics 239: 107280.

Tu W and Pindera MJ (2013) Targeting the finitedeformation response of wavy biological tissues with bioinspired material architectures. Journal of the Mechanical Behavior of Biomedical Materials 28: 291-308.

Wang X, Pan E, Albrecht JD, et al. (2009) Effective properties of multilayered functionally graded multiferroic composites. Composite Structures 87(3): 206-214.

Xiao JH, Xu YL and Zhang FC (2016) Generalized selfconsistent electroelastic estimation of piezoelectric nanocomposites accounting for fiber section shape under antiplane shear. Acta Mechanica 227(5): 1381-1392.

Yang H, Abali BE, Timofeev D, et al. (2020) Determination of metamaterial parameters by means of a homogenization approach based on asymptotic analysis. Continuum Mechanics and Thermodynamics 32: 1251-1270.

$\mathrm{Yu}$ Li J and Dunn ML (2001) Variational bounds for the effective moduli of heterogeneous piezoelectric solids. Philosophical Magazine A 81(4): 903-926.

Zhang T, Zhang K and Liu W (2019) Exact impact response of multi-layered cement-based piezoelectric composite considering electrode effect. Journal of Intelligent Material Systems and Structures 30(3): 400-415.

Zhu J, Bettaieb MB and Abed-Meraim F (2020) Numerical investigation of necking in perforated sheets using the periodic homogenization approach. International Journal of Mechanical Sciences 166: 105209.

\section{Appendix}

The multiphysics finite-element method developed for comparison with the FVDAM predictions follows the same zeroth-order homogenization framework, cf. Chen and Wang (2020). That is, the displacement and electric potential in the $q$ th element are represented by a two-scale expansion involving macroscopic and fluctuating contributions:

$$
\begin{aligned}
& u_{i}^{(q)}(\mathbf{x}, \mathbf{y})=\bar{\varepsilon}_{i j} x_{j}+u_{i}^{\prime}(q)(\mathbf{x}, \mathbf{y}) \\
& \alpha^{(q)}(\mathbf{x}, \mathbf{y})=-\bar{E}_{j} x_{j}+\alpha^{\prime(q)}(\mathbf{x}, \mathbf{y})
\end{aligned}
$$

The fluctuating parts of the displacement and electric potential in each element are approximated using the Q8-type interpolation function and nodal quantities:

$$
u_{i}^{\prime}(q)=\sum_{p=1}^{8} N_{p}(\eta, \xi) u_{i}^{o(p, q)}, \alpha^{\prime(q)}=\sum_{p=1}^{8} N_{p}(\eta, \xi) \alpha^{o(p, q)}
$$

where $u_{i}^{o(p, q)}$ and $\alpha^{o(p, q)}$ are the nodal displacements and electric potential. The index $p$ indicates the node number at the four corners and four midpoints of the actual element starting from the lower-left corner and processing counterclockwise.

The employed two-scale expansion of displacements and electric displacements produces local strain and electric field in the form:

$$
\boldsymbol{\varepsilon}^{(q)}(\mathbf{x}, \mathbf{y})=\overline{\boldsymbol{\varepsilon}}+\mathbf{B}_{u}^{(q)}{\mathbf{\mathbf { u } ^ { \prime }}}_{o}^{(q)}, \mathbf{E}^{(q)}(\mathbf{x}, \mathbf{y})=\overline{\mathbf{E}}-\mathbf{B}_{\varphi}^{(q)} \boldsymbol{\varphi}_{o}^{(q)}
$$

where $\mathbf{u}_{o}^{(q)}$ and $\boldsymbol{\varphi}_{o}^{\prime(q)}$ are vectors including the fluctuating nodal displacements and electric potentials, respectively. $\mathbf{B}_{u}^{(q)}$ is the standard strain-displacement matrix of the $q$ th element while $\mathbf{B}_{\varphi}^{(q)}$ represents the gradient vector relating the electric-field and electric potentials. For a piezoelectric solid within a domain $V$, the total potential energy of the system is the summation of bulk strain and electric energies and the work done by the external force and electric charge:

$$
\begin{aligned}
\pi & =U^{b}+U^{e}-W \\
& =\frac{1}{2} \int_{V}\left(\boldsymbol{\sigma}^{\mathrm{T}} \boldsymbol{\varepsilon}-\mathbf{D}^{\mathrm{T}} \mathbf{E}\right) d V-\left(\overline{\boldsymbol{\sigma}}^{\mathrm{T}} \overline{\boldsymbol{\varepsilon}}-\overline{\mathbf{D}}^{\mathrm{T}} \overline{\mathbf{E}}\right)
\end{aligned}
$$

Using the generalized Hooks' law, equation (A4) can be further expressed as:

$$
\begin{aligned}
\pi & =U^{b}+U^{e}-W \\
& =\frac{1}{2} \int_{V}\left[(\mathbf{C} \boldsymbol{\varepsilon}-\mathbf{e} \mathbf{E})^{\mathrm{T}} \boldsymbol{\varepsilon}-\left(\mathbf{e}^{\mathrm{T}} \boldsymbol{\varepsilon}+\mathbf{\kappa} \mathbf{E}\right)^{\mathrm{T}} \mathbf{E}\right] d V \\
& -\left(\overline{\boldsymbol{\sigma}}^{\mathrm{T}} \overline{\boldsymbol{\varepsilon}}-\overline{\mathbf{D}}^{\mathrm{T}} \overline{\mathbf{E}}\right)
\end{aligned}
$$

Substituting equation (A3) into equation (A5) and making the first variation yields:

$$
\begin{aligned}
\delta \pi= & \delta \mathbf{u}_{o}{ }_{o}^{\mathrm{T}}\left(\mathbf{K}_{u u} \mathbf{u}_{o}^{\prime}+\mathbf{K}_{u \varphi} \boldsymbol{\varphi}_{o}^{\prime}-\mathbf{f}_{u}\right) \\
& +\delta \varphi^{\prime}{ }_{o}^{\mathrm{T}}\left(\mathbf{K}_{u \varphi}^{\mathrm{T}} \mathbf{u}_{o}^{\prime}+\mathbf{K}_{\varphi \varphi} \boldsymbol{\varphi}_{o}^{\prime}{ }_{o}-\mathbf{f}_{\varphi}\right)
\end{aligned}
$$

where $\mathbf{K}_{u u}=\int_{V} \mathbf{B}_{u}^{\mathrm{T}} \mathbf{C} \mathbf{B}_{u} d V, \mathbf{K}_{\varphi \varphi}=\int_{V} \mathbf{B}_{\varphi}^{\mathrm{T}} \mathbf{C} \mathbf{B}_{\varphi} d V, \mathbf{f}_{u}=$ $\int_{V}\left(\mathbf{B}_{u}^{\mathrm{T}} \mathbf{e}^{\mathrm{T}} \overline{\mathbf{E}}-\mathbf{B}_{u}^{\mathrm{T}} \mathbf{C} \varepsilon\right) d V$ and $\mathbf{f}_{\varphi}=\int_{V}\left(-\mathbf{B}_{\varphi}^{\mathrm{T}} \mathbf{e} \varepsilon-\mathbf{B}_{\varphi}^{\mathrm{T}} \mathbf{\kappa} \overline{\mathbf{E}}\right) d V$. The global system of equations is obtained by $\delta \pi=0$ :

$$
\left[\begin{array}{ll}
\mathbf{K}_{u u} & \mathbf{K}_{u \varphi} \\
\mathbf{K}_{u \varphi}^{\mathrm{T}} & \mathbf{K}_{\varphi \varphi}
\end{array}\right]\left[\begin{array}{l}
\mathbf{u}_{o}^{\prime} \\
\boldsymbol{\varphi}_{o}^{\prime}
\end{array}\right]=\left[\begin{array}{l}
\mathbf{f}_{u} \\
\mathbf{f}_{\varphi}
\end{array}\right]
$$

